\title{
Application of Light Scattering to Polymers, Liquid Crystals, and Biological Systems
}

\author{
Hyuk Yu \\ Department of Chemistry, University of Wisconsin, Madison, WI 53706
}

August 10, 1981

\begin{abstract}
The applications of elastic and quasielastic light scattering techniques to polymers in dilute solution, thermotropic liquid crystals and biological membrane vesicles are presented. It is focused on how we extract specific structural features or dynamic processes of these condensed medium samples through the light scattering methods. The paper reviews the light scattering studies that were carried out at the author's laboratory in Wisconsin from 1973 until 1980.
\end{abstract}

Key words: amphoteric latex; dynamic light scattering; light scattering; nematic liquid crystal; photo-receptor membrane vesicles, random coil polymers.

\section{Introduction}

With the advent of the dynamic light scattering method and the attendant detection techniques, [1-5], we are now able to expand the application of light scattering methods to a variety of diverse condensed medium systems hitherto unexplored. In this paper, I will outline the kinds of light scattering methods that we use in my laboratory at Wisconsin to probe the structure and dynamics of polymers, nematic liquid crystals, and biological membranes. Four scattering techniques shall first be described with appropriate examples in each case, and then I will move on to the studies of (a) amphoteric latex system, (b) photo-receptor disk membrane vesicles, (c) binary nematic solution, and (d) intrachain dynamics of random coil polymers, in order to bring home the power and limitation of various light scattering techniques. At the outset I must emphasize that this paper is not intended as an exhaustive review of the state-ofthe art of light scattering methods, but rather as a report of how one academic research laboratory uses these methods to explore diverse problems of interest.

\section{Elastic Light Scattering}

Extensive treatises have been written on the subject such that I need not dwell on the historic overview or lengthy explication of the technique. Instead, I will focus on a rather

\footnotetext{
' Figures in brackets indicate literature references at the end of this paper.
}

narrowly defined problem of how to determine the linear dimension of scattering particles with substantial symmetry in shape, particularly when the linear dimension $R$ is comparable to the incident wavelength $\lambda$. The problem of this kind arises for example in trying to determine the large radius of gyration [9] of $T$ even bacteriophage DNA whose molecular weights are on the order of $10^{8}$ daltons. If one tries to effect the customary procedure of the Zimm plot [10] to extract the radius of gyration, the scattering intensity profile must be obtained at such small angles that one encounters substantial technical problems [9]. By small angle scattering, I mean that one must obtain the data in the Guinier region, [11] i.e., $Q R \leq 1$ where $Q$ is the momentum transfer. We propose a new scheme to determine the linear dimension $R$ when the scattering particles are so large that $Q R \leq 1$ is difficult to attain experimentally. The method is to focus on the structure of scattering profile at higher angles where the Bragg condition is fulfilled, i.e., $Q R=$ $m / \pi$ where $m$ is an integral multiple constant. To be more specific, I quote some simple examples such as solid sphere, hollow sphere, spheroidal shape of either oblate or prolate axial ratio, and the corresponding shell structures. The isotropic parts of the particle form factor in the limit of Rayleigh-Gans-Debye scattering [12] of some of these are given below.

a. Solid sphere

$$
P(\theta)=\left[j_{0}(x)+j_{2}(x)\right]^{2}
$$


where $x \equiv Q R, R$ is the radius and $j_{k}(x)$ is the $k$ th order spherical Bessel function.

\section{b. Hollow sphere $[13,14]$}

$$
P(\theta)=\left[\frac{3}{x^{3}\left(1-l^{3}\right)}(\sin x-\sin x l-x \cos x+x l \cos x l)\right]^{2}
$$

where $x \equiv Q R, l \equiv a / R, R$ and $a$ are respectively the outer and inner radii.

\section{c. Ellipsoids of revolution [15]}

$$
P(\theta)=\frac{9 \pi}{2} \int_{0}^{2 \pi} \frac{J_{3 / 2}^{2}(u)}{u^{3}} \cos \beta d \beta
$$

where $u \equiv Q a\left(\cos ^{2} \beta+\frac{b^{2}}{a^{2}} \sin ^{2} \beta\right)^{1 / 2}, a$ and $b$ are semi-major and semi-minor axes, $\beta$ is the angle between the semi-major axis and bisectrix, and $J_{3 / 2}$ is the $3 / 2$ th order Bessel function.

\section{d. Circular cylinder}

$P(\theta)=\frac{\pi}{2 v} \int_{0}^{\pi / 2} \frac{1}{\cos \beta}\left[J_{1 / 2}(v \cos \beta) \cdot \frac{2 J_{1}(u \sin \beta)}{u \sin \beta}\right]^{2} \sin \beta d \beta(4)$

where $a$ and $l$ are the radius and length of the cylinder, $u \equiv$ $Q a, v \equiv Q / 2$, and $\beta$ is the angle between the cylinder axis and bisectrix.

\section{e. Spheroidal shells}

$$
\begin{aligned}
P(\theta) & =\int_{0}^{1} \frac{\sin ^{2}\left[x \sqrt{1-q t^{2}}\right]}{x^{2}\left(1-q t^{2}\right)} d t \\
& =\int_{0}^{1} \frac{\sin ^{2}\left[y\left(\frac{1-q t^{2}}{1-q}\right)^{1 / 2}\right]}{y^{2}\left(\frac{1-q t^{2}}{1-q}\right)} d t
\end{aligned}
$$

for oblate shell where $x \equiv Q b, y=Q a, q \equiv 1-\left(\frac{\mathrm{a}}{\mathrm{b}}\right)^{2}$, and $b$ and $a$ are respectively the semi-major and semi-minor axes such that $0 \leq q \leq 1$.

$$
\begin{aligned}
\mathrm{P}(\theta) & =\int_{0}^{1} \frac{\sin ^{2}\left[x \sqrt{1+p t^{2}}\right]}{x^{2}\left(1+p t^{2}\right)} d t \\
& =\int_{0}^{1} \frac{\sin ^{2}\left[y\left(\frac{1+p t^{2}}{1+p}\right)^{1 / 2}\right]}{y^{2}\left(\frac{1+p t^{2}}{1+p}\right)} d t
\end{aligned}
$$

for prolate shell where $x$ and $y$ are the same as above, $a$ and $b$ are respectively the semi-major and semi-minor axes, and $p \equiv(a / b)^{2}-1$ such that $0 \leq p \leq \infty$.

As an illustration of the proposed method, we take the simplest structure, i.e., sphere, and show how the Bragg condition is extracted from which the radius of sphere is deduced. The isotropic particle form factor of sphere in the
Rayleigh-Gans-Debye limit is given by the square of the sum of the zeroth and second order spherical Bessel function as in eq (1), and its structure is a monotonically decreasing function from unity at zero scattering angle to $x=\tan x$ where it gives the first minimum. This is illustrated in figure 1 where a semi-logarithmic plot of the particle form factor $P(\theta)$ versus sine of one-half of the scattering angle $\theta$ is given for spherical particles having the radii of 100,110 , 120 , and $130 \mathrm{~nm}$ suspended in water and scattered by incident blue light of $436 \mathrm{~nm}$ in wavelength. It is clear from the plots that under these conditions the radius must exceed $120 \mathrm{~nm}$ for $P(\theta)$ to give the first minimum; $P(\theta)$ is fairly structureless for the radius less than $120 \mathrm{~nm}$. If, on the other hand, we plot $(Q R)^{4} P(\theta)$ against $\sin (\theta / 2)$, the graphs as shown in figure 2 result. This arises because the damping profile of

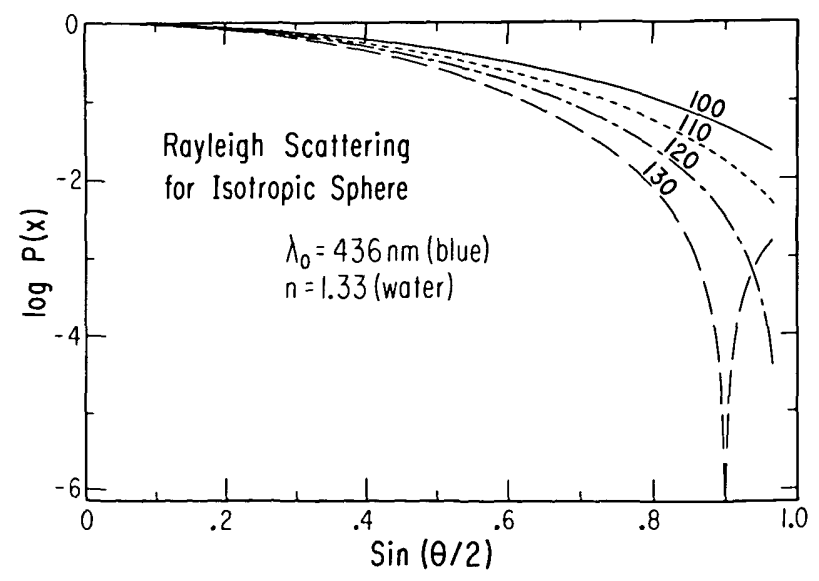

FIGURE 1. The isotropic part of the particle form factor $P(x)$ of Rayleigh-Gans-Debye scattering from solid, isotropic spheres against $\sin (\theta / 2)$ for the radius of $100,110,120$ and $130 \mathrm{~nm}$, with the incident wavelength of $436 \mathrm{~nm}$ (blue) in a medium with the refractive index $\mathrm{n}$ of 1.33 (water). Note that $x \equiv Q R$ hence $P(x)=P(\theta)$.

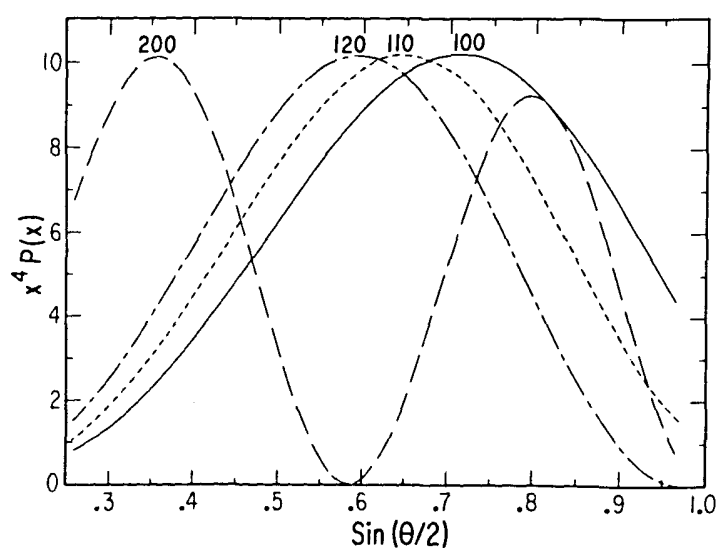

FICURE 2. $x^{4} P(x)$ versus $\sin (\theta / 2)$ of figure 1 . In place of $R=130 \mathrm{~nm}$, plot for $200 \mathrm{~nm}$ is shown. 
$P(\theta)$ in eq $(1)$ is compensated by the factor $(Q R)^{4}$. Thus, the monotonic descrease of $\mathrm{P}(\theta)$ for small particles is rendered to have a maximum by multiplying by $(Q R)^{4}$. Algebraically this is easily seen from eq (1);

$$
x^{4} P(\theta)=9\left(\frac{\sin x}{x}-\cos x\right)^{2}
$$

where $x \equiv Q R=\frac{4 \pi n}{\lambda_{0}} \sin (\theta / 2) \cdot R$ and the extrema positions of $P(\theta)$ appear at

$$
\tan x=\frac{x}{1-x^{2}} \text { for maxima }
$$

and

$$
\tan x=x \text { for minima }
$$

The plots in figure 2 are drawn for the common experimental scattering conditions in mind, namely the scattering angle spans from $30^{\circ}$ to $150^{\circ}$. Experimentally the ordinate scale is immaterial to the extent that one is interested in determining the extrema positions to extract the particle radius. For completeness sake, we show in figures 3 and 4 the analogous set of plots for spherical particles with longer radii as though they could be analyzed by the RayleighGans-Debye approximation, whereas its applicability is limited [8] in this size range, and $\mathrm{P}(\theta)$ itself contains sufficient structure that $x^{4} P(\theta)$ plot affords at best sharper maxima and smoother minima. I shall later return to the limit of applicability of Rayleigh-Gans-Debye scattering relative to the particle size. We display in figure 5 why the factor $x^{4}$ is chosen as the multiplication factor to $P(\theta)$ in order to moderate its damping at higher angles. For a given radius of a particle, we show three profiles whereby it is made clear that a factor $x^{2}$ does not reduce the damping sufficiently,

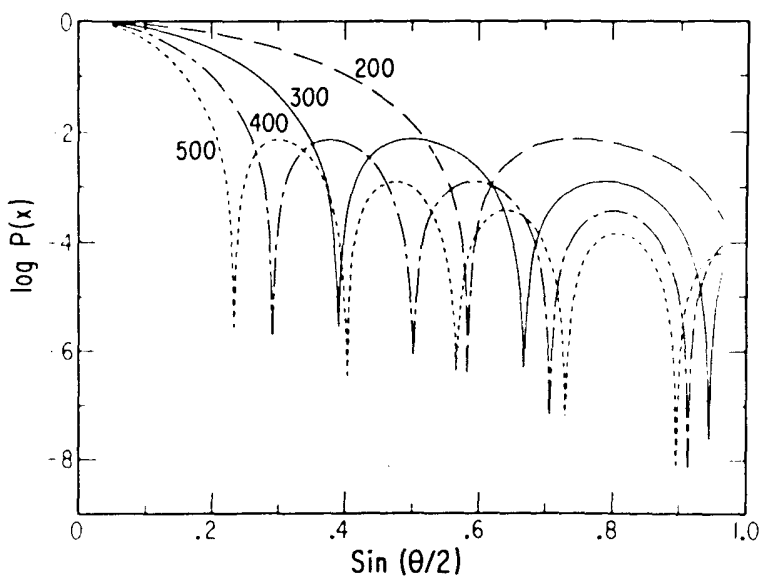

Figure 3. Similar to figure 1 with $R=200,300,400$ and $500 \mathrm{~nm}$. whereas a factor $x^{6}$ overcompensates the damping. The optimum is clearly the $x^{4}$ factor.

Turning to the experimental verification of the proposed method, [16], we show in figure 6 a plot of $I_{v v}(\theta) \cdot \sin ^{4}(\theta / 2)$ versus $\sin (\theta / 2)$ for a polystyrene latex standard (Polysciences, Lot 2-1435), whose radius is given as $87 \pm 4 \mathrm{~nm}$ determined by transmission electron microscope, suspended in distilled water. Here, the subscripts $v v$ of the scattered intensity stand for the polarized scattering, namely the polarizer and analyzer are both oriented vertically relative to the scattering plane. We should note that the ordinate scale is arbitrary and $x^{4} P(\theta)$ is proportional to $I_{v v}(\theta) \cdot \sin ^{4}(\theta / 2)$, since $P(\theta)$ is the isotropic part of the particle form factor. The data points were taken at $1^{\circ}$ increments and the solid curve was drawn according to eq (9) with $\lambda_{0}=$ $436, n=1.333, R=80 \mathrm{~nm}$. The discrepancy of 10 percent between the radius of $87 \mathrm{~nm}$ by electron microscopy and that of $80 \mathrm{~nm}$ by this method may be ascribed to a number of artifacts arising from electron beam optics in the electron microscopy technique. In figure 7, we show a test of another

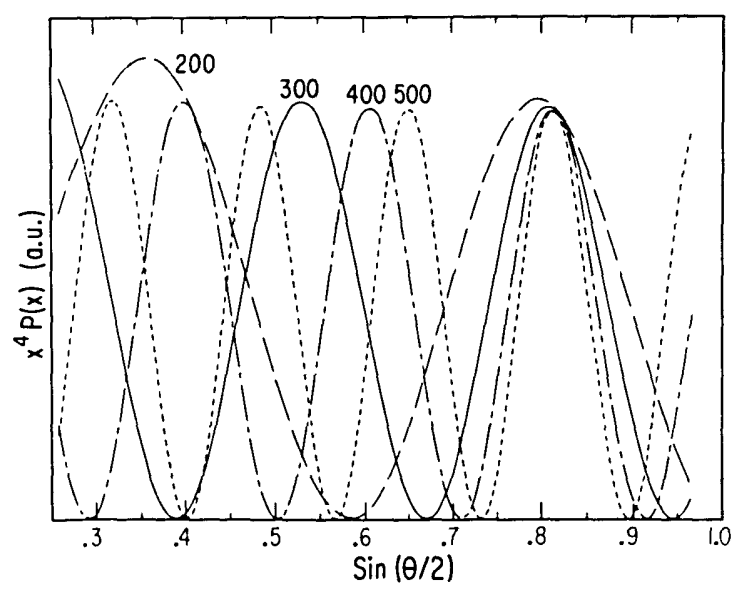

FIGURE 4. Similar to figure 2 with $R=200,300,400$ and $500 \mathrm{~nm}$.

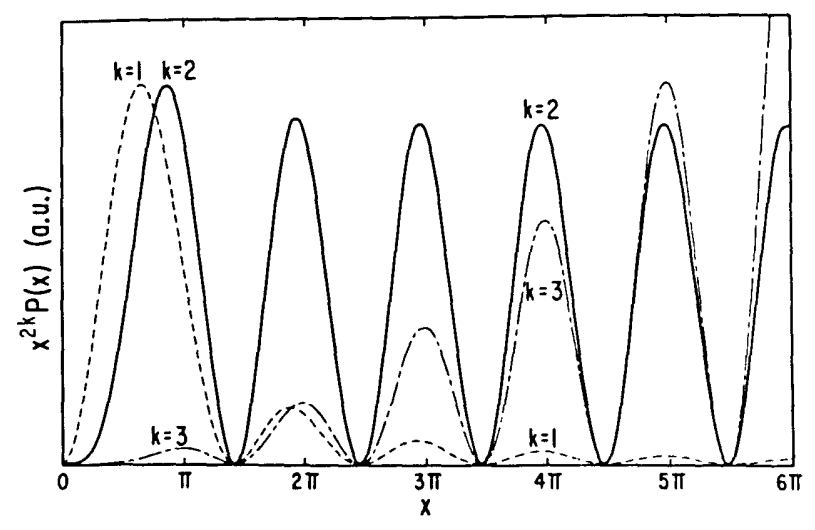

FIGURE 5. $x^{2} P(\theta)$ and $x^{6} P(\theta)$ versus $x$ 
polystyrene latex standard (Polysciences, Lot 2380), having the radius of $139 \mathrm{~nm}$ again determined by electronic microscopy. Two curves are drawn for comparison. The first (solid curve) is that predicted by the Mie scattering function with $R=136 \mathrm{~nm}$ and the other (dashed curve) is that predicted by eq (9) with the same $R$. In the Mie function fitting, we use the refractive index ratio $m$ of the particle to medium as 1.21 [17]. In the inset, the scattering profiles at different concentrations of the latex particles are shown where the concentration range of the most (a) to the least (d)

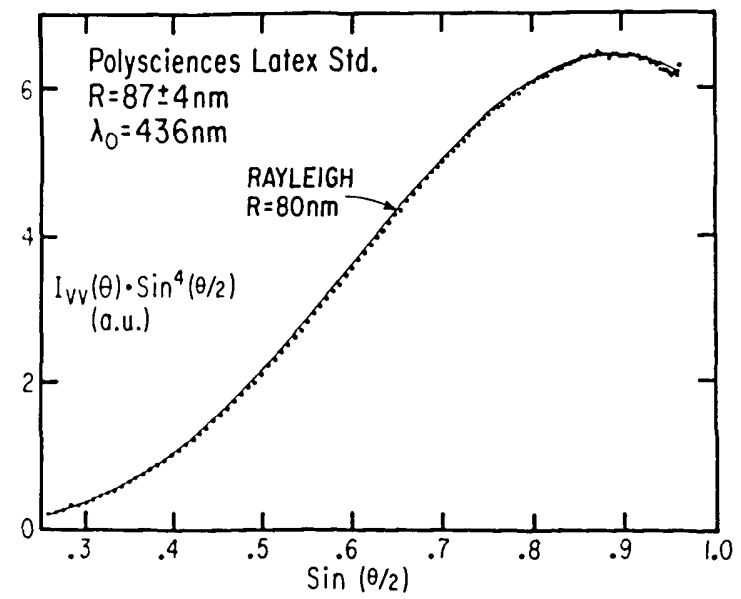

Figure 6. Calibration run of the light scattering method with Polysciences latex standard with $R=87 \pm 4 \mathrm{~nm}$ (mean $\pm \mathrm{s}$.d.). The scattering intensity here is observed with the incident and scattered beams vertically polarized relative to the scattering plane. The ordinate is scaled in arbitrary units, and the solid curve represents the Rayleigh-Gans-Debye scattering function for an isotropic solid sphere with $80 \mathrm{~nm}$ raduis.

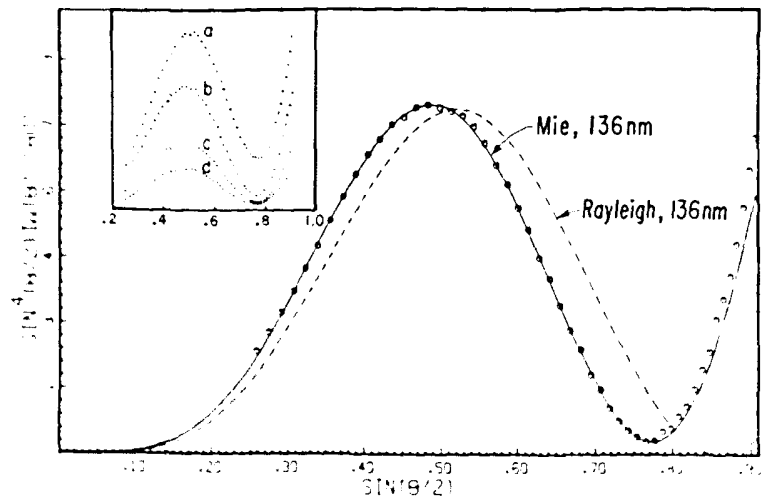

Ficure 7. Calibration run with Polyscience latex standard with $R=135 \pm$ $1 \mathrm{~nm}$. Experimental conditions are identical with those in Figure 6. Dashed curve is drawn with the Rayleigh scattering function with $R=136 \mathrm{~nm}$ and solid curve with the Mie function with $R=136 \mathrm{~nm}$. The inset shows the concentration dependence of scattering profile whereby a represents the most concentrated and $d$ the least concentrated. The number density of latex particles in a is about one order of magnitude larger than ind. concentrated is about one order of magnitude. It shows well that the scattering profiles are weakly dependent on the concentration, and we could have deduced the radius of 130 $\mathrm{nm}$ from the most concentrated case (a) shown in the inset. The least concentrated case (d) is expanded in the plot of figure 7 . Ignoring the matching with the entire scattering profile but focusing on the maximum and minimum positions inferred from eq (9) for the Rayleigh-Gans-Debye scattering, we would have deduced the radius of $144 \mathrm{~nm}$. Thus for this size particle, either scattering function would have sufficed if the radius determination within 4 percent is acceptable. It should however be noted that the discrepancy of the radius determinations by the two scattering functions exceeds the precision limit of extrema position determination in this size range.

Having thus established that our proposed method works for the radius determination of a spherical particle of $R \leq$ $140 \mathrm{~nm}$ suspended in aqueous media, we now turn to the applicability limit of Rayleigh-Gans-Debye scattering and when one must use the Mie scattering function. In order to stipulate the applicability limit, we compare the extrema positions predicted from eq (9) and those computed from the Mie functions. The latter computation was performed on a Harris/7 computer. The comparison is provided in figures $8(\mathrm{~A})$ and $8(\mathrm{~B})$ where the reduced size parameter $\alpha$, defined as $2 \pi R \lambda$, is given in terms of the extrema positions in the scattering angle of $x^{4} P(\theta)$ profiles for the polarized and unpolarized scatterings respectively. When $m=$ 1.0001 , we recover the predictions of the Rayleigh-GansDebye scattering as given by eq (9); this particular value of $m$ is not significant as long as $m$ is very close but still larger than unity for the Mie function to be evaluated. These figures allow us to understand why the radius deduced from the Rayleigh function always overestimates. At any given extreme position, be it a maximum or minimum, $\alpha$ values for $m>1$ invariably lie below that for $m=1$ although the relative error committed by assuming $m \approx 1$ is not a monotonically increasing function of $m$ for any size particles. This is particularly true with the maximum positions which show oscillation with respect to $m$. Hence, the relative error should also oscillate. In fact, we can evaluate the error. This is illustrated in figure $9(A)$ and $9(B)$ where the percent error of analyzing the scatterng profile extrema according to the Rayleigh-Gans-Debye scattering is plotted against the reduced size parameter $\alpha$ at different values of $m$. The polarized and unpolarized scattering cases are shown in (A) and (B), respectively, In either case, the error can be equal or less than 10 percent if $m \leq 1.15$ for $\alpha \leq 7$. The oscillation in the error estimate becomes progressively larger in amplitude as the $m$ value increases. For the values of $m$ close to unity, the error with respect to the particle size is sensibly constant though with a slight increase with $\alpha$ in each $m$. Hence, one should not approximate with impunity 
the scattering of small particles by the Rayleigh scattering function regardless of the refractive index ratio $m$.

In closing this subject, let me emphasize that the spherical particle size analysis can be effected by determinations of the extrema positions of $\sin ^{4}(\theta / 2) I(\theta)$ profiles and these are provided by analytical solutions of

$$
\begin{gathered}
x=\tan x \quad(\text { minima) } \\
\frac{x}{1-x^{2}}=\tan x \quad \text { (maxima) }
\end{gathered}
$$

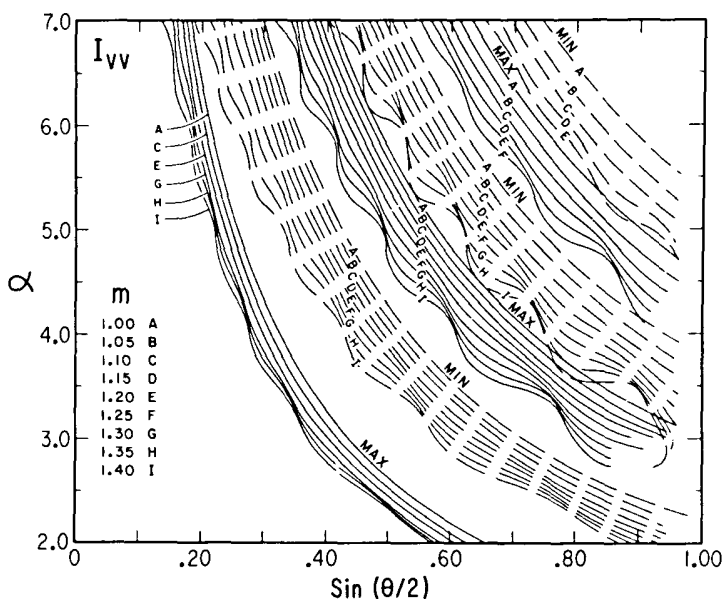

FIGURE 8A. Ratio of isotropic solid sphere's circumference to wavelength in scattering medium, $\alpha \equiv 2 \pi R / \lambda$, versus $\sin (\theta / 2)$ at different extrema positions of the Mie function for $x^{4} P(\theta)$. Each set is drawn at different refractive index ratios $m$. The maxima and minima are distinguished by solid and dashed curves respectively. This is for the Mie function at the vertical/vertical optical configuration.

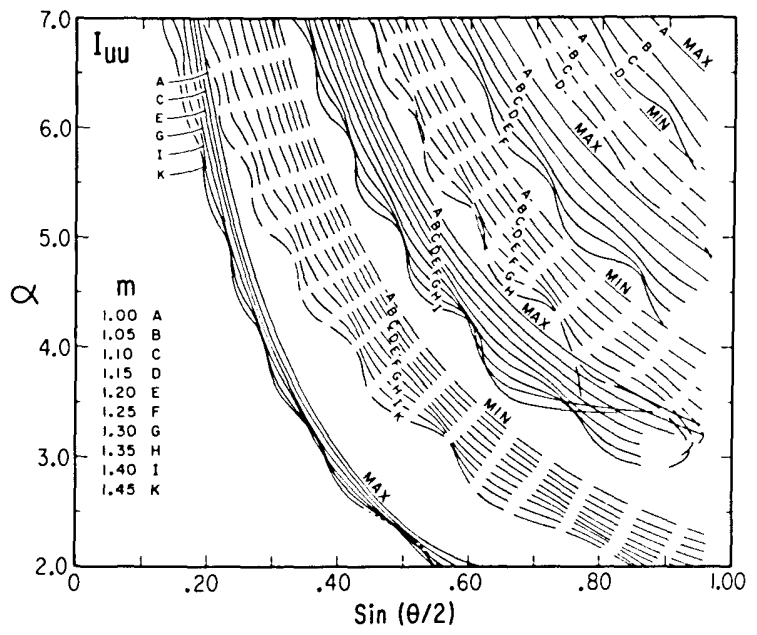

Figure 8B. Analogous to (8a) for the case of Mie function at unpolarized/unpolarized configuration.

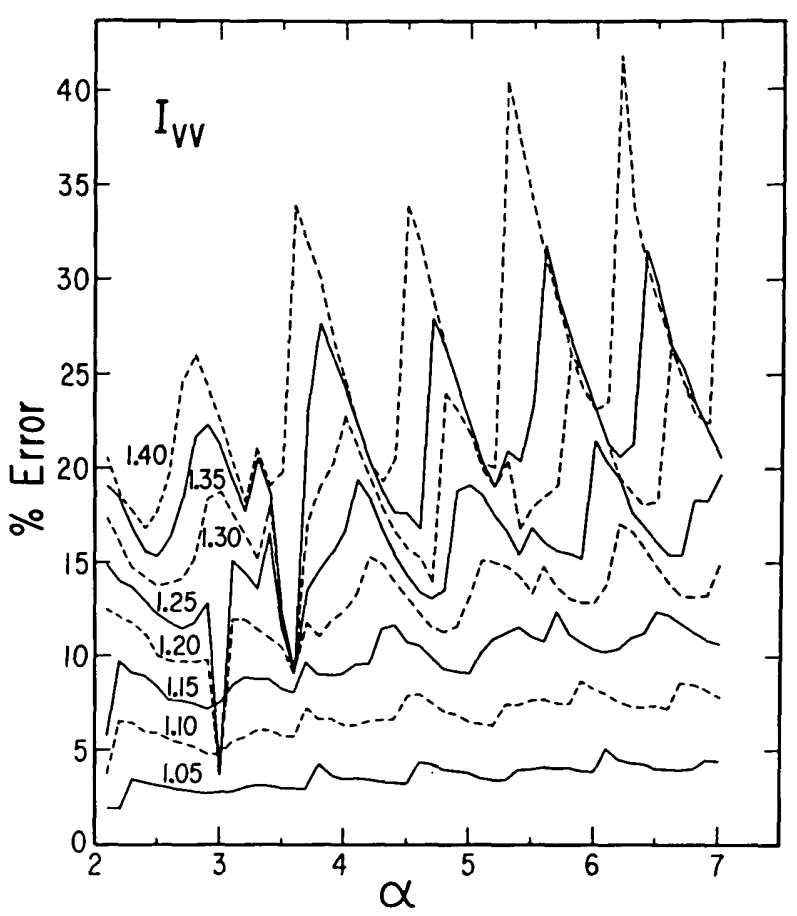

FIGURE 9A. Percent error of analyzing the profile extrema positions according to Rayleigh-Gans-Debye scattering as a function of the reduced size parameter $\alpha$ at different refractive index ratios $m$, for the polarized scattering.

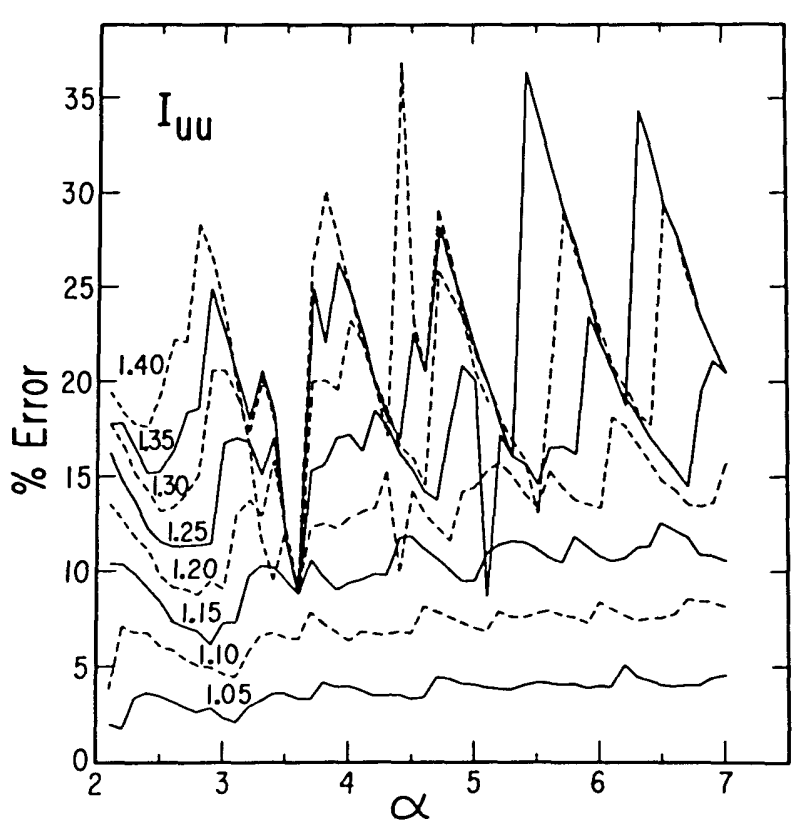

FIGURE 9B.Analogous to (9a) for the unpolarized scattering. 
in the case of Rayleigh-Gans-Debye scattering whereas they must be evaluated numerically in the case of Mie scattering. Because the method depends on the structure of scattering function at high $Q$, it is restricted to monodisperse systems and extrapolation to infinite dilution is not an essential step in the procedure provided the scattering suspension is dilute enough.

\section{Quasielastic Light Scattering}

Here, we restrict our discussion to the thermally induced, spontaneous concentration fluctuations whereby the translational diffusion coefficient of the scattering particles at infinite dilution is deduced. The homodyne power spectrum [1] $S(Q, v)$ of the Doppler broadened scattering from a monodisperse system of particles is

$$
S(\mathbf{Q}, \nu)=A \frac{\Delta \nu_{1 / 2}}{\nu^{2}+\left(\Delta \nu_{1 / 2}\right)^{2}}+B
$$

where $A$ is an optical constant which depends on the intensity factor of the spectrum, $B$ is a constant, a measure of shot-noise level and the spectral half-width at half-height $\Delta \nu_{1 / 2}$ is related to the translational diffusion coefficient $D$ by

$$
\Delta \nu_{1 / 2}=D Q^{2} / \pi
$$

Our instrument [18] is schematically depicted in a block diagram in figure 10. A typical power spectrum obtained from a Dow Polystyrene Latex standard suspension (45.4 $\mathrm{nm}$ radius) is shown in figure 11 and the corresponding spectral halfwidth against $Q^{2} / \pi$ is displayed in figure 12 where the scattering angle spans the range $10^{\circ}-100^{\circ}$. The diffusion coefficient deduced via eq (11) is $(5.39 \pm 0.04) \times$ $10^{-8} \mathrm{~cm}^{2} / \mathrm{s}$ which is in turn converted to the Stokes radius of $45.5 \pm 0.4 \mathrm{~nm}$. Progression of the $\mathrm{S} / \mathrm{N}$ ratio of the observed power spectrum with the number of accumulations is shown in figure 13.

\section{Electrophoretic Light Scattering}

The technique was first developed by Ware and Flygare in 1971 [19] and subsequently by Uzgiris in 1972, [20] and it has since undergone substantial refinements [21-29]. The

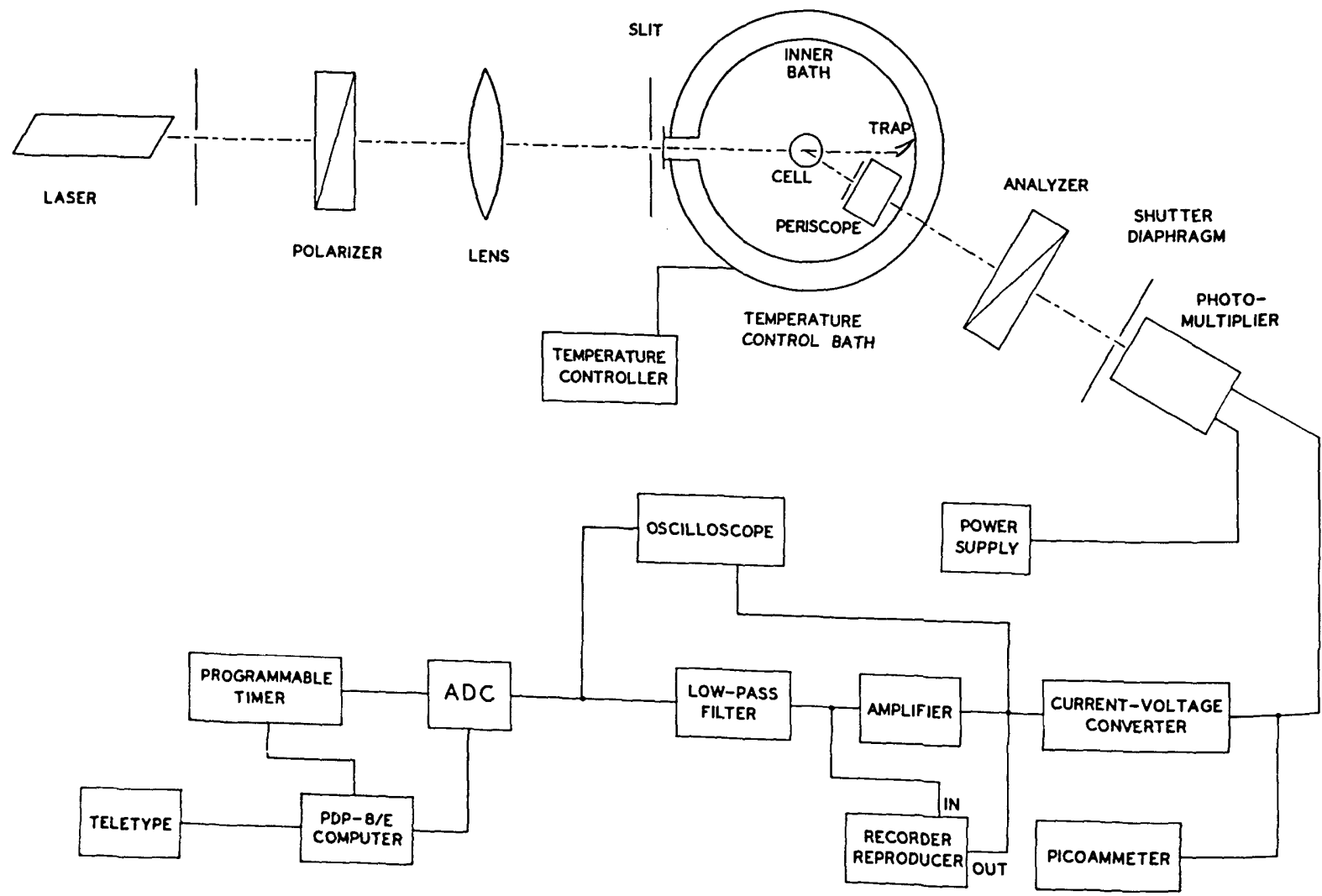

FIGURE 10. Block diagram of Rayleigh spectrometer. 
advantages that it offers over the conventional electrophoresis methods have now been well-documented in the literature $[25,27]$. As with any new technique, this one also had to first be calibrated against those of more conventional methods with the use of a test system. Ware and Flygare chose bovine serum albumin (BSA) for the purpose because it was one of the best characterized globular proteins and commercially available in a relatively pure form. Others have subsequently chosen BSA for the same reason to calibrate their instruments [28-32].

The method is no more than another application of laser velocimetry. A monodisperse system of charged particles in dilute solution under the influence of an applied electric field would drift uniformly to the oppositely charged electrode. The diffusion equation governing this situation [4] in

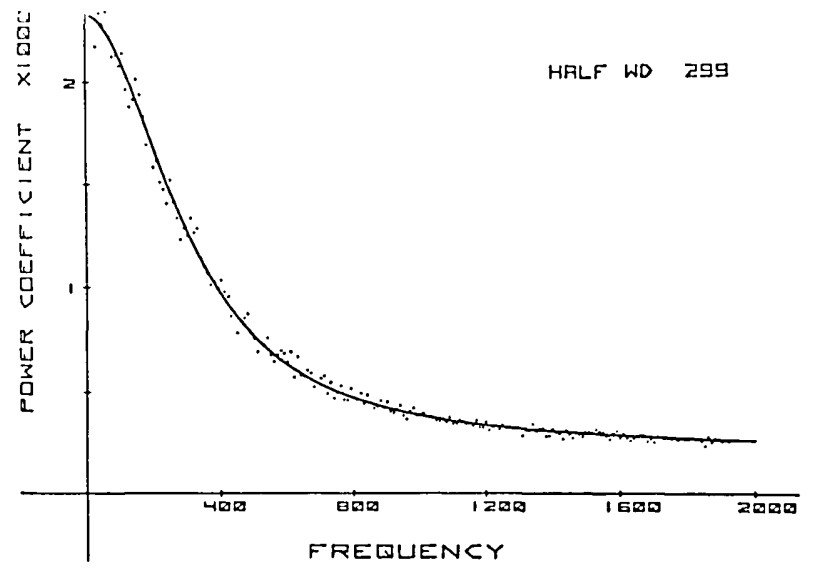

FIGURE 11. Homdyne power spectrum of a polystyrene latex standard at 2 $\mathrm{kHz}$ bandwidth with $\Delta v_{1 / 2}=299 \mathrm{~Hz}$.

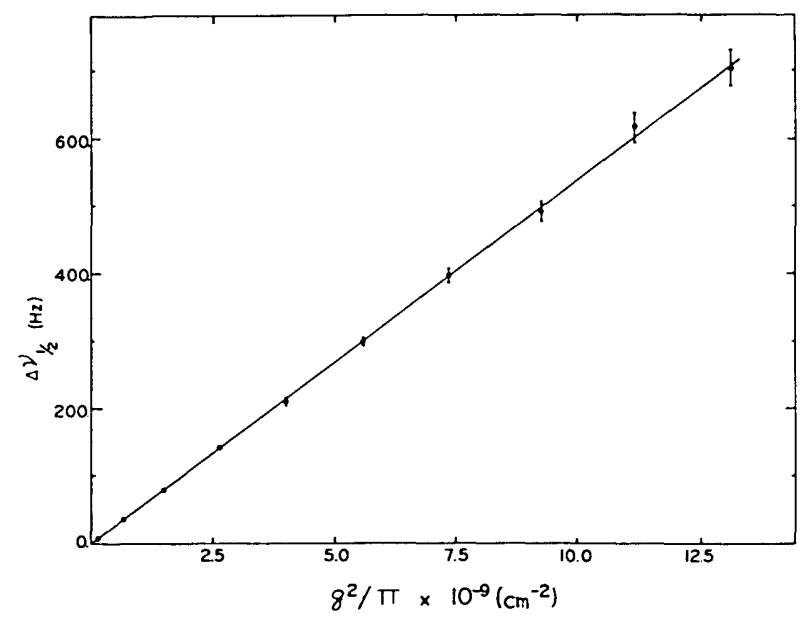

FigURE 12. $\Delta v_{0}$ versus $q^{2} / \pi$ whose slope yields $D=(5.39 \pm 0.04) \times$ $10^{-12} \mathrm{~m}^{2} / \mathrm{s}$ and the Stokes raduis of $45.6 \pm 0.4 \mathrm{~nm}$. terms of the self part of the space-time autocorrelation function $G_{s}(\mathbf{R}, t)$ is

$$
\frac{\partial}{\partial t} G_{s}(\mathbf{R}, t)+\mathbf{V}_{d} \cdot \nabla G_{s}(\mathbf{R}, t)=D \nabla^{2} G_{s}(\mathbf{R}, t)
$$

with the initial condition

$$
G_{s}(\mathbf{R}, 0)=\delta(\mathbf{R})
$$

where $\mathbf{V}_{d}$ is the uniform drift velocity, $\mathrm{D}$ the translational diffusion coefficient of particle and $\delta(\mathbf{R})$ is the Dirac delta function. Upon taking the space Fourier transforms of the above, we have

$$
\frac{\partial}{\partial t} F_{s}(\mathbf{Q}, t)+i \mathbf{Q} \cdot \mathbf{V}_{d} F_{s}(\mathbf{Q}, t)=-Q^{2} D F_{s}(\mathbf{Q}, t)
$$

with

$$
F_{s}(\mathbf{Q}, 0)=1
$$

The solution of eq (14) with the initial condition, eq (15), is

$$
F_{s}(\mathbf{Q}, t)=e^{-\mathbf{Q}^{2} D t} e^{i \mathbf{Q} \cdot v_{d} t}
$$

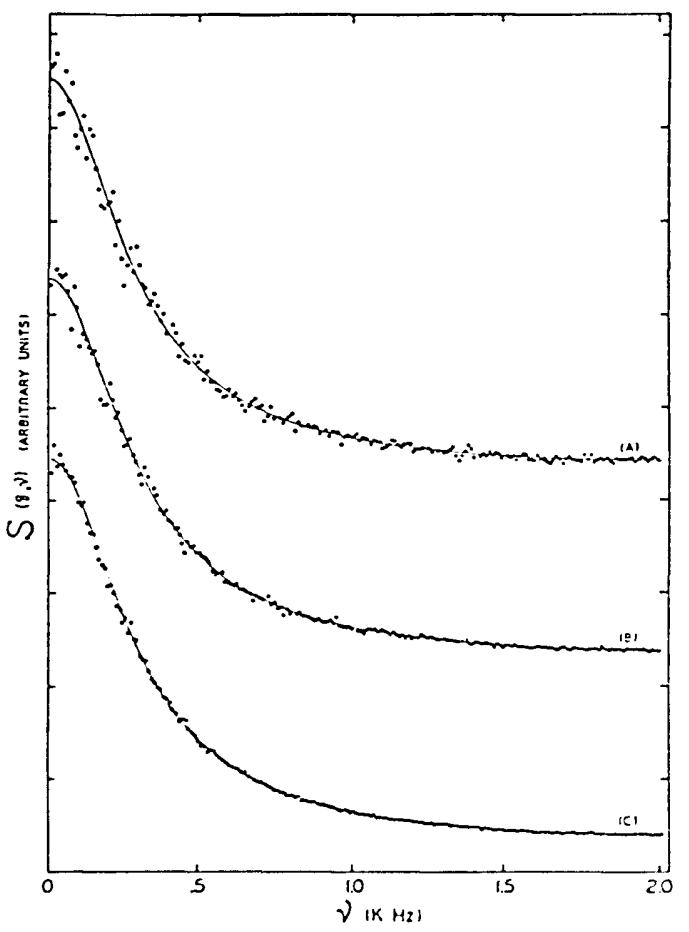

Figure 13. Power spectra of polystryene latex standard taken at $60^{\circ}$ scattering angle. A, B, and C are 250,1000 and 4000 scan averages, respectively. 
The power spectrum of heterodyne beating from a system of charged particles governed by eq (16) is a Doppler shifted Lorentzian whose shifted angular frequency $\Delta \omega_{s}$ is given by

$$
\Delta \omega_{s}=\mathbf{Q} \cdot \mathbf{V}_{d}
$$

Our scattering geometry is represented in figure 14. In order to effect heterodyne beating, the scattering angle has to be fairly small $\left(2^{\circ}-8^{\circ}\right)$ so as to make use of cell surface reflected light as the local oscillator component. Thus the shifted frequency

$$
\Delta \nu_{s}=Q V_{d} \cos (\theta / 2) / 2 \pi \approx \pi n \mu\left(\theta V_{p p}\right) / 360 d \lambda_{\circ}
$$

where $n$ is the refractive index of scattering medium, $\mu$ the electrophoretic mobility (the drift velocity per unit field strength), $\theta$ the scattering angle, $V_{p p}$ the peak-to-peak applied voltage, $d$ the electrode spacing ( $1.84 \mathrm{~mm}$ in our case) and $\lambda_{0}$ the incident wavelength in vacuo. Hence, the shift frequency $\Delta \nu_{s}$ should be proportional to the product, $\theta V_{p p}$, and the electrophoretic mobility $\mu$ can be deduced by determining $\Delta v$, measured at different scattering angles and $V_{p p}$.

Since there were some discrepancies in the mobility values of BSA reported by Ware and Flygare $[19,21]$ and Mohan et al. [28] by electrophoretic light scattering and those by Schlessinger [30], Alberty [31], and Longsworth and
Jacobsen [32] with the moving boundary method, we have set out to examine the discrepanices [33]. The purpose was to test the accuracy attainable by this technique vis-a-vis that of a more conventional method. In so doing, we have not only found the mobility by this technique to be in com. plete accord with those of the moving boundary method but also established that the BSA mobility depends on the ionic strength of the suspending medium according to Henry's formulation $[34,35]$. The latter finding is neither without parallel nor unexpected [36-38], but the ease of the experiment to confirm it points to the utility and power of the technique. At the same time, our experiment, which covers a wide range of ionic strength, points to certain limitations and the complementary nature of the technique with more conventional methods.

The discrepancies referred to above were entirely due to the sample polydispersity and had little bearing on the veracity of the electrophoretic light scattering technique. This was confirmed by examining the two sets of samples. First is the so-called Fraction V of Armour (Lot A21505) which was used by Ware and Flygare as well as by all others with the moving boundary method, and the other is the BSA Monomer Standard of Pentex brand from Miles Laboratories. Polyacrylamide gel electrophoresis patterns of the two are compared in figure 15 . In figure 16 , we display how well the shifted frequency depends linearly on the product $\left(\theta \cdot V_{\text {app }}\right)$ for the Fraction $V$ sample. Finally we compare the deduced mobilities, corrected to $20^{\circ} \mathrm{C}$ in water, of the two samples relative to their ionic strength dependence in figure 17. In the same figure, we plot all other available

GEOMETRY OF ELECTROPHORETIC SCATTERING

Applied Field: along the $z$-axis
Light Scattering: on the $x-y$ plane
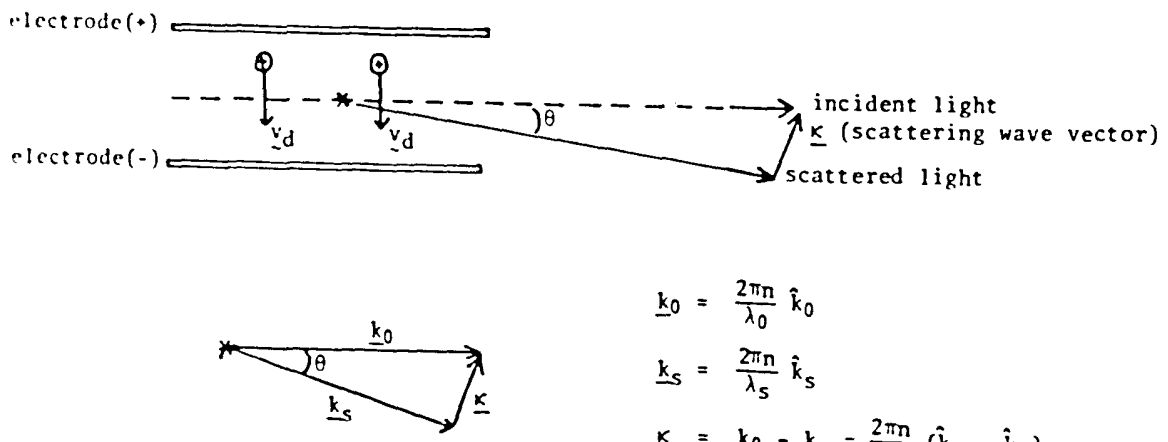

$$
\begin{aligned}
\underline{k}_{0}= & \frac{2 \pi n}{\lambda_{0}} \hat{k}_{0} \\
\underline{k}_{s}= & \frac{2 \pi n}{\lambda_{s}} \hat{k}_{s} \\
\underline{k}= & \underline{k}_{0}-\underline{k}_{s}=\frac{2 \pi n}{\lambda_{0}}\left(\hat{k}_{0}-\hat{k}_{s}\right) \\
& \text { where } \lambda_{0}=\lambda_{s} \text { (quasiclastic) }
\end{aligned}
$$

FIGURE 14. Scattering geometry of electrophoretic light scattering, where the symbol $\mathrm{K}$ is used in place of $Q$. 


\section{Armour Fraction $\mathrm{V}$}
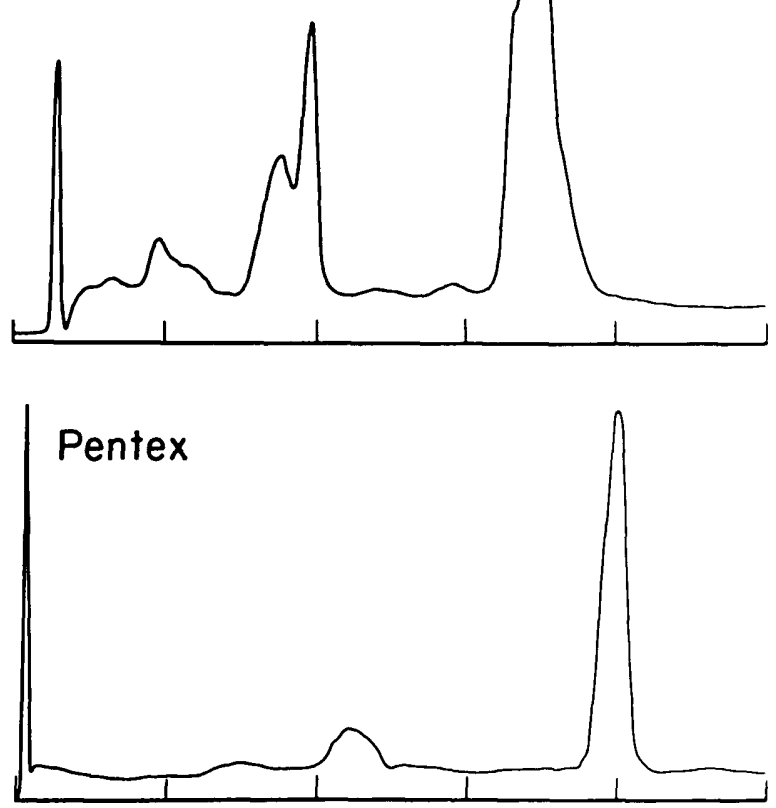

FIGURE 15. Polyacrylamide gel electrophoresis patterns of polydisperse BSA Fraction V of Armour and Pentex brand BSA monomer standard.

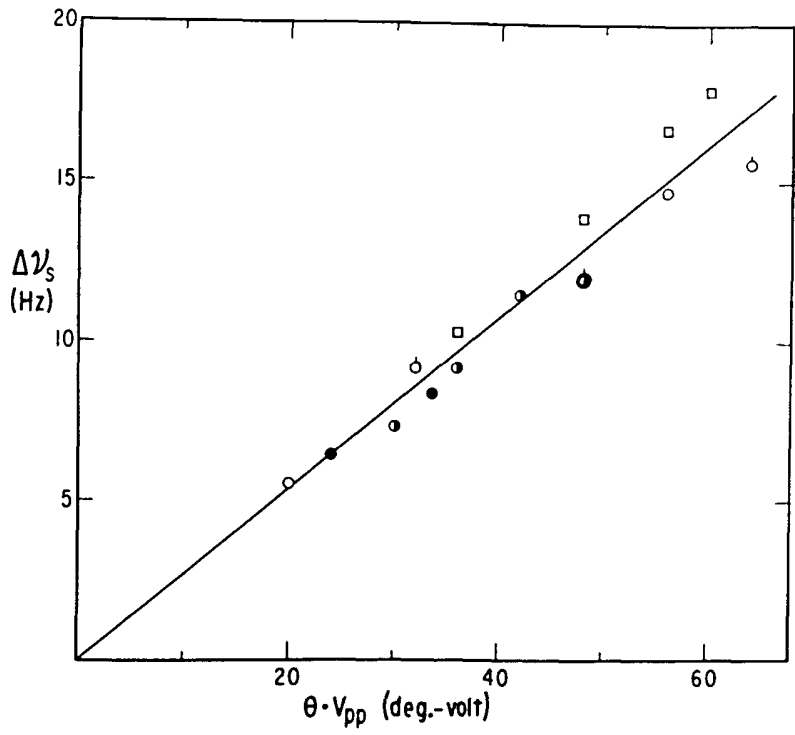

FICURE 16. Electrophoresis Doppler shift frequency $\Delta \nu_{s}$ versus $\theta V_{p p}$ for Fraction $\mathrm{V}$ samples at $5 \mathrm{mM}$ ionic strength. Three independently prepared samples are examined. Scattering angles of two preparations are distinguished as (O) $2^{\circ},(O) 3^{\circ},(\bigcirc) 4^{\circ},(O) 4^{\circ}$ and third preparation is measured at $(\square) 6^{\circ}$.
BSA data in the literature at $\mathrm{pH}$ 8.7-9.4 after making appropriate temperature correction. Two solid curves are drawn according to Henry's equation

$$
\mu=\frac{Q_{E}}{6 \pi \eta R} f(\varkappa R)
$$

where $Q_{E}$ is the net charge of the electrokinetic unit, $\eta$ the viscosity of the medium, $R$ the radius of the unit, $x^{-1}$ is the Debye screening length, and $f(x R)$, which accounts for the ionic strength dependent charge screening, is given by

$$
\begin{aligned}
f(\varkappa R)=(1+\varkappa R)^{-1}\{ & 1+\frac{(\varkappa R)^{2}}{16}-\frac{5(\varkappa R)^{3}}{48}-\frac{(\varkappa R)^{4}}{96}+\frac{(\varkappa R)^{5}}{96} \\
+ & {\left.\left[\frac{(\varkappa R)^{4}}{8}-\frac{(\varkappa R)^{6}}{96}\right] e^{x R} \int_{x R}^{\infty} t^{-1} e^{-t} d t\right\} }
\end{aligned}
$$

where the integral in the last term is the exponential integral $E_{1}(\varkappa R)$ [39]. With use of the Stokes radius of $36 \AA$ deduced from the translational diffusion coefficient of BSA by Baldwin et al. [40] and with the dependence of $x^{-1}$ on ionic strength $I$ at $20{ }^{\circ} \mathrm{C}$ as $x^{-1}=3.045 / \sqrt{I(\AA)}$, the two curves are drawn with 20 and 26 electronic charges per BSA molecule for $Q_{E}$, respectively, for the lower (Fraction V) and upper (Pentex) sets. Since the data by the moving boundary method are all obtained with the Fraction $\mathrm{V}$ samples, it is

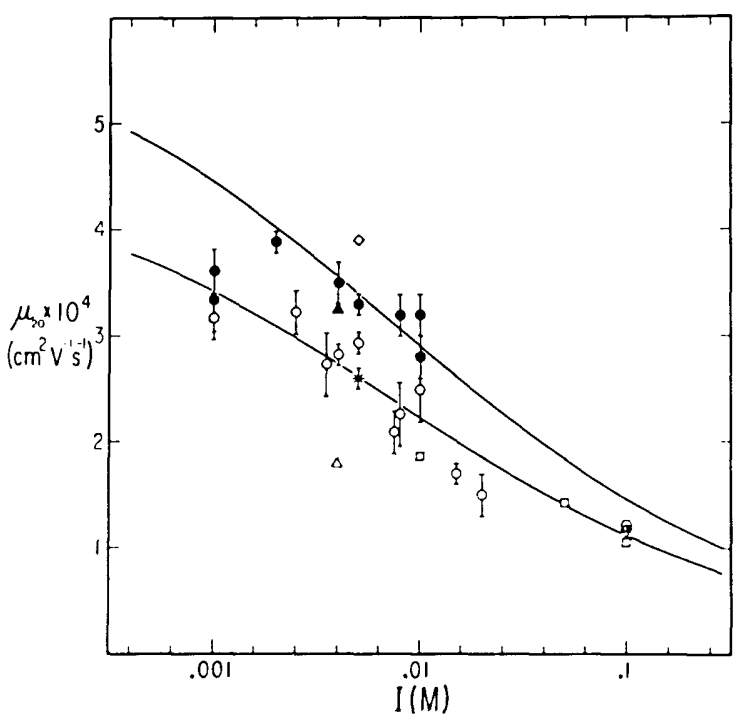

FIGURE 17. Electrophoretic mobility, corrected to $20^{\circ} \mathrm{C}$ in water, versus ionic strength. Open circles with error bars are Fraction $\mathrm{V}$ sample, filled circles with error bars are Pentex sample. ${ }^{*}$ is from the slope of figure 16. All others are from the literature; $(\square)$ Schlessinger, (O) Alberty, $(O$ and $\nabla)$ Longsworth and Jacobsen, $(\Delta) W$ are and Flygare [20] and ( $\Delta) W$ are and Flygare 22 and $(\diamond)$ Mohan et al. [29] 
not surprising that they belong to the lower set and are consistent with ours. The results in figure 17 represent the first instance, to the best of our knowledge, of a systematic study of BSA mobility dependence on ionic strength at a given pH. It is clear that Henry's equation can well account for the ionic strength dependence of electrophoretic mobility.

\section{Forward Depolarized Scattering (FDS)}

The technique was first proposed and utilized by Wada and coworkers in 1969-70 [41, 42] for the determination of the rotatory diffusion coefficient of a rod-like molecule, i.e., tobacco mosaic virus. A further refinement was reported by Schurr and Schmitz in 1973 with the same system [43] and an extension to calf-thymus DNA followed [44]. I will illustrate the technique with use of an example, namely the intrachain dynamics of linear flexible macromolecules in dilute solution. A theoretical formulation of the spectral profile of a scattered optical field is provided by 0 no and Okano. We have slightly generalized the scheme by concluding that the FDS spectrum is a superposition of uniformly weighted multiple Lorentzian as long as there exists the normal coordinate transformation for the intramolecular chain dynamic modes [45].
A model of dilute polymer solution is composed of optically isotropic solvent and linear flexible chains, each of which is constituted of $n+l$ anisotropic elements with cylindrical symmetry. The FDS spectral profile is formally given by

$$
I_{V_{h}}(\omega)=B(N / V) \int_{-\infty}^{\infty}<\sum_{i=0}^{n} \sum_{j=0}^{n} \alpha_{x y}^{(i)}(\tau) \alpha_{x y}^{(j)}(0)>e^{-i \omega \tau} d \tau
$$

where $B$ is an optical constant, $(N / V)$ is the number density of polymer molecules within the scattering volume, the subscripts $V$ and $h$ refer to the vertical and horizontal polarization directions of the incident and scattered optical fields, respectively, and $<>$ refer to the equilibrium ensemble average. If the normal coordinate transformation is possible, the long wavelength modes in hydrodynamic regime is

$$
I_{V_{k}}(\omega)=B(N / V)(\Delta \alpha / 3)^{2} \sum_{k=1}^{n} \frac{1 / \tau_{k}}{\omega^{2}+\left(1 / \tau_{k}\right)^{2}}
$$

where $\Delta \alpha$ is the optical anisotropy of each element and $\tau_{k}$ is the kth normal mode relaxation time. In case of a monodisperse system of dilute rigid rod molecules, the heterodyne power spectrum corresponding to eq (2l) is

$$
S_{\perp}(0, \nu)=A \frac{(1 / 2 \pi \tau)}{\nu^{2}+(1 / 2 \pi \tau)^{2}}
$$
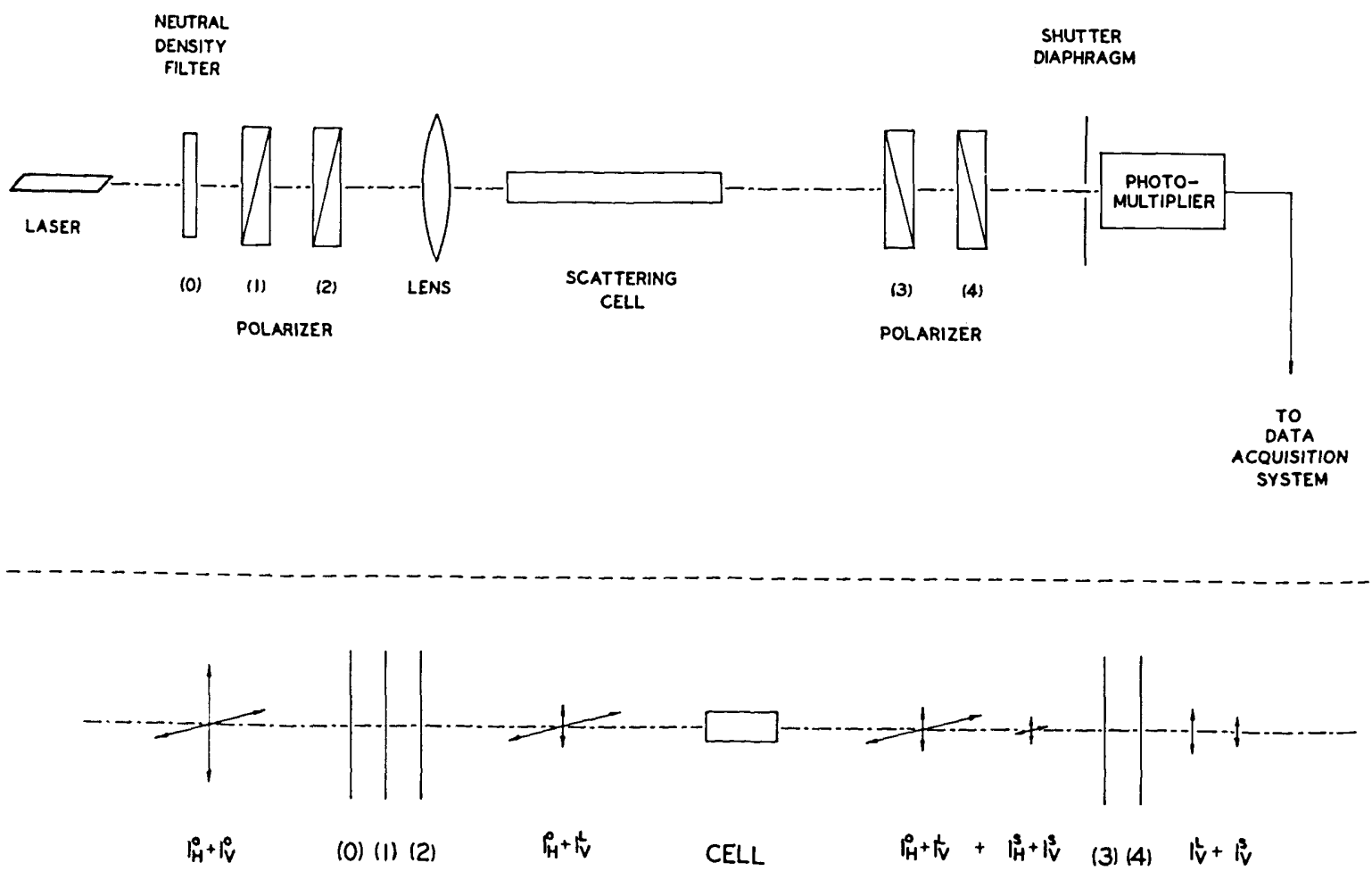

FICURE, 18. Block diagram of the Rayleigh spectrometer in the FDS configuration and the filter train. Here, $I^{\circ}, I^{P}$ and $F$ stand for the incident, leakage and scattering intensities, respectively, and the subscripts $V$ and $H$ designate vertical and horizontal polarization directions. 
where $\tau$ is the relaxation time of rotatory diffusion of the rod.

The optical train of our apparatus [46] is shown in figure 18 , and an example of $S_{\perp}(0, \nu)$ for a dilute solution of poly(n-hexyl isocyanate), a rigid rod chain of $3500 \AA$ in length, is given in figure 19.

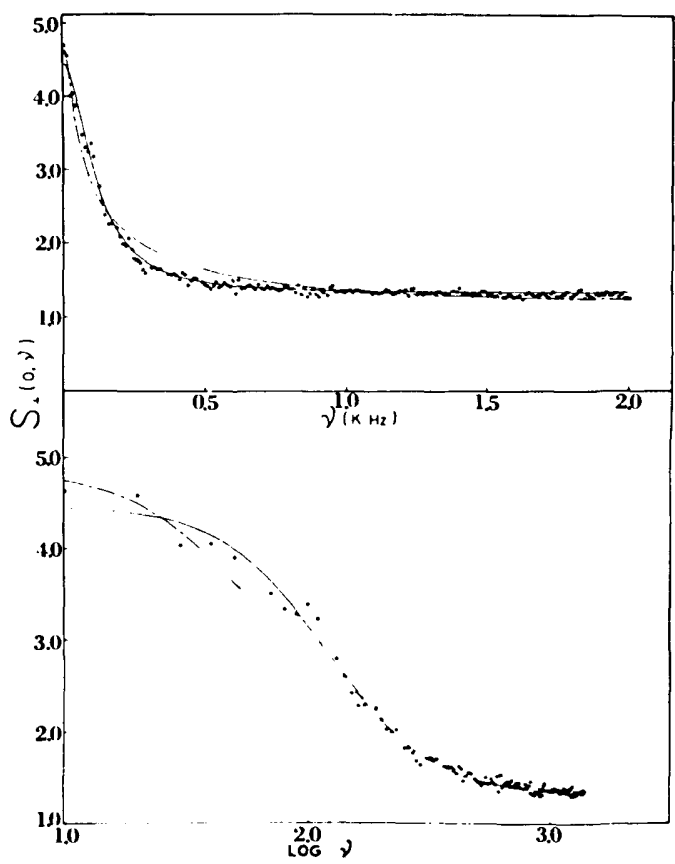

FIGURE 19 FDS power spectrum of PHIC (M.W. $3.2 \times 20^{5}$ ) in n-hexane at $\mathrm{C}=2.16 \mathrm{mg} / \mathrm{mL}$ with single Lorentzian fit $(-)$ and $Z_{\text {imm spacing fit }}$ $(-\cdot-)$. Upper and lower graphs are distinguished by linear and logarithmic frequency scales.

The four light scattering techniques that we employ to study a variety of scattering systems are summarized above. Before leaving this section, I note parenthetically that our data acquisition and analysis system makes use of a minicomputer (PDP8/e) and a set of two microprocessor computers (Apple II) interacting with a Harris/7 system. This is shown schematically in figure 20 where only the part dealing with computer controlled automatic goniometer on SOFICA light scattering photometer is yet to be implemented while all others are now in operation.

\section{Systems}

Turning to the four systems that were studied by one or more of the above techniques, table I summarizes what we were able to learn about these systems by the light scattering methods. For the sake of brevity, I will not discuss all of them in the same detail but rather highlight some selected aspects of some of them.

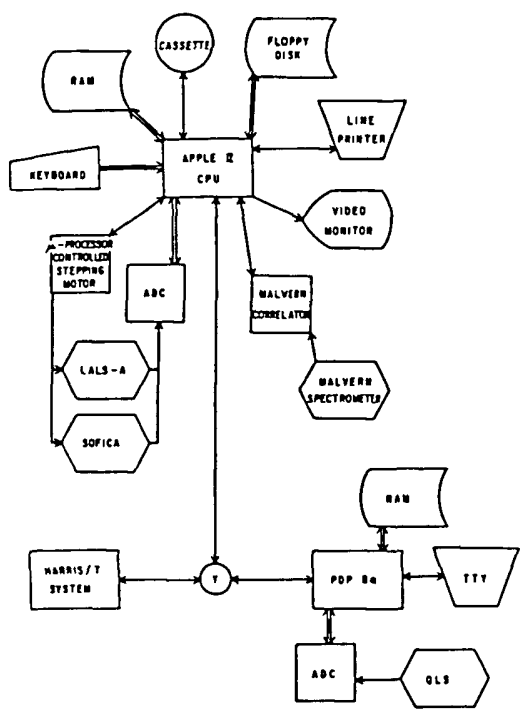

FIGURE 20. Data acquisition and analysis system of quasielastic light scattering in time domain (Malvern autocorrelator) and in frequency domain (PDP 8/e) and those of elastic light scattering.

\section{a. Amphoteric Latex Particles}

Monodisperse latex particles have been utilized in a wide variety of fields including immunochemical assays [47, 48] and biomembrane studies [49] in addition to their more conventional applications as the markers and calibration standards in microscopy and light scattering and as the model colloids [50]. Our interest coincides with the last instance where an amphoteric latex system with the well-defined number of charges can be invoked as a model for globular proteins and biomembrane vesicles relative to the ionization behavior of their surface groups. Recently, Homola and James [51] were able to prepare an amphoteric latex system without any added surfactants which now meets the requirement of a well-defined charge number. The latex particles consist of three monomers, styrene (S), methacrylic acid (MA), and N,N-diethylaminoethyl methacrylate (DEAM), emulsion polymerized with persulfate at $70^{\circ} \mathrm{C}$.

We were mainly interested in whether there exists any size change at different $\mathrm{pH}$ [52]. One of the transmission electron micrographs taken of the samples is shown in figure 21 which makes it evident that the sample has a relatively homogeneous size distribution. Results of the conductometric and potentiometric titrations, as shown in figure 22, give clear evidence that the latex particles are indeed amphoteric in nature and the titration valences at the extreme $\mathrm{pH}$ of 3 and 11 are fairly symmetric at about $6 \times 10^{5}$ electronic charges per particle. This was deduced from the titration results and the size determination, which was performed by elastic light scattering according to the method 
TABLE 1. List of the four systems studied by light scattering techniques

\begin{tabular}{cccc}
\hline SYSTEM & TECHNIQUES & STRUCTURE or PROPERTIES & VARIABLES \\
\hline Amphoteric Latex Particles & ELS & Surface Layer Dilation; & $\mathrm{pH}$ \\
& QLS & electrostatic interactions & \\
& EPLS & & \\
\end{tabular}

Photoreceptor

Retinal Disc Membranes

\section{ELS}

QLS

EPLS
Optical Birefringence

NMR Line Splittings

FDS
1. osmotic deformation

2. membrane lateral stiffness

3. ion permeability

4. surface ion binding

$\begin{array}{llll}\text { Nematic Liquid Crystals } & \text { Optical Birefringence } & \text { 1. Orientation Distribution } & \text { 1. non-nematogen composition } \\ & \text { NMR Line Splittings } & \text { Function } & \text { 2. temperature } \\ \text { FDS } & \text { 2. order fluctuation dynamics } & \end{array}$

Linear Macromolecules

FDS

internal normal modes

ELS: elastic light scattering-total scattered intensity profile

QLS: quasielastic light scattering-angular dependent spectral shape

EPLS: electrophoretic light scattering-electrical field dependent QLS

FDS: forward depolarized scattering

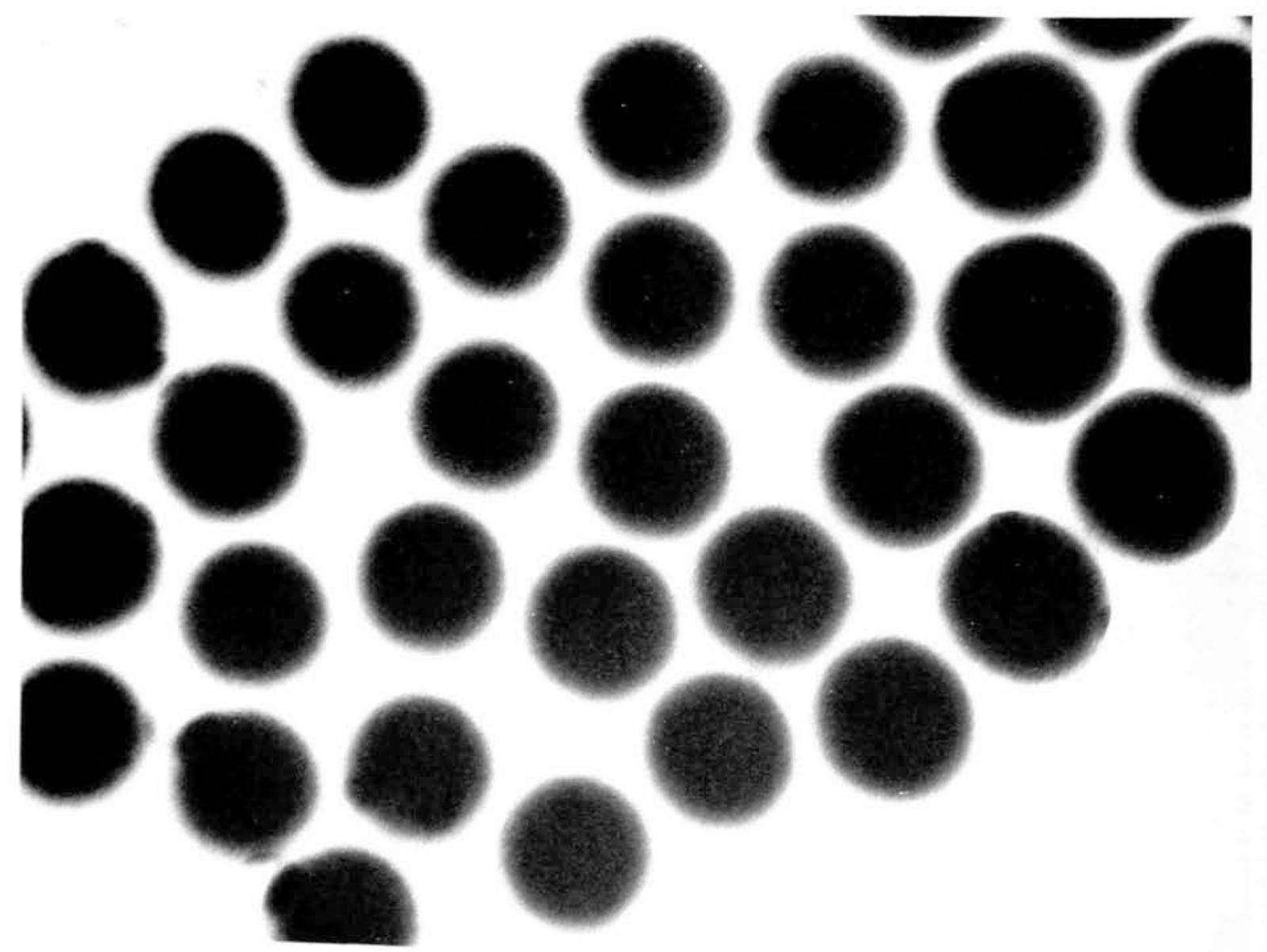

FiGURE. 21. Transmission electron micrograph of amphoteric latex particles at $\mathrm{X}_{67,000}$ 


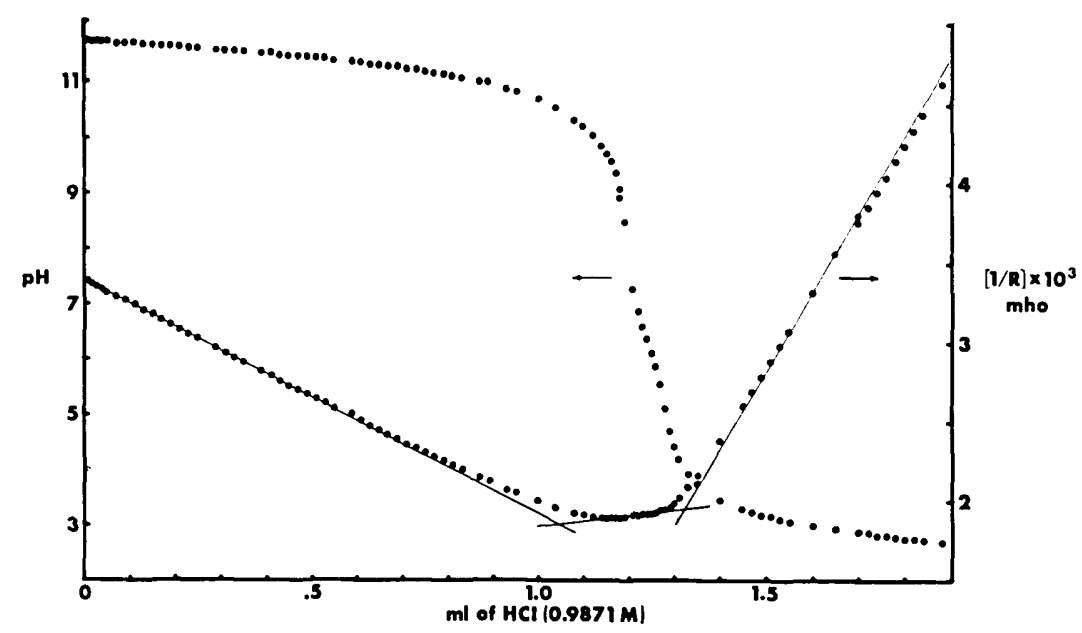

FIGURE 22. An example of potentiometric and conductometric titration. For the conductometry, the left intersection point of extrapolated lines is taken as the starting point of protonation of amino groups and the right intersection point as the termination point of protonation of carboxylate ions.

outlined earlier. In figures 23 through 25 , we show the scattering profiles, plotted according to eq (9), at nominal $\mathrm{pH}$ of 3,7 and 11 , all at the same ionic strength of $1 \mathrm{mM}$. We display in figure 26 all the radius data so obtained at different $\mathrm{pH}$ from 3 to 11 at the same ionic strength. It is evident that there is a symmetric size change with respect to $\mathrm{pH}$ and the point of minimum radius at around $\mathrm{pH} 7$ coincides with the isoelectric point determined by titration. The Stokes radii obtained by quasielastic light scattering agree with those in figures 23 and $25,125 \pm 3 \mathrm{~nm}$ and $123 \pm 3 \mathrm{~nm}$ respectively at $\mathrm{pH} 3$ and 11 , while that of $118 \pm 2 \mathrm{~nm}$ at $\mathrm{pH} 7$ does not agree with the radius in figure $24,112 \pm 2 \mathrm{~nm}$. Collecting all these results, we propose a model as depicted schematically in figure 27. It consists of hydrophobic core mainly constituted of styrene monomer and of hydrophilic shell made predominantly of ionic comonomers, MA and DEAM. The observed size change is then attributed to the chain expansion in the shell layer due to electrostatic repulsive interactions while the core remains relatively intact with respect to $\mathrm{pH}$ changes in the suspending medium. What remains uncertain however is the difference between the Stokes radius and that determined by elastic light scattering at $\mathrm{pH}$ 7. Whether the difference could be attributed to the thickening of the hydration layer at the isoelectric point must be examined by another technique such as NMR.

Before closing I must remark that one could raise the issue of whether our scheme of size determination is indeed probing the outer radius of swollen latex particles as contrasted to some ill-defined average of the inner and outer radii. After performing a set of simulation studies with concentric spheres having different segment densities in the shell volume relative to that in the core, we are convinced that our method is likely to underestimate the outer radius

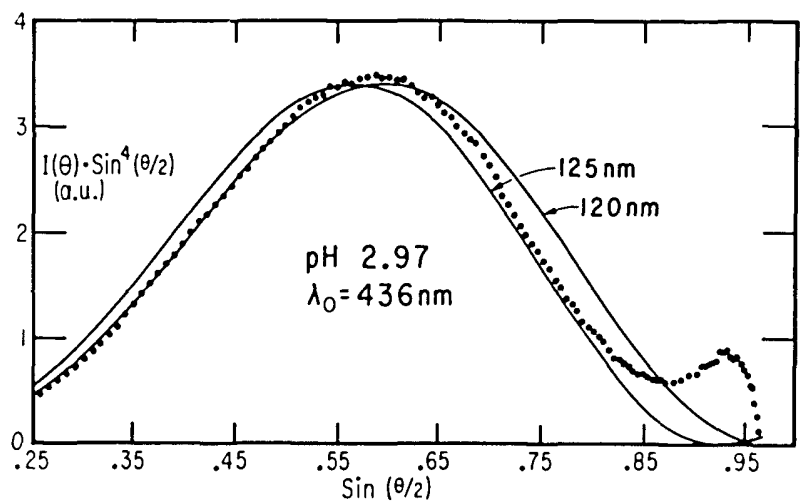

FIGURE 23. Light scattering profile of amphoteric latex at $\mathrm{pH} 2.97$ where the polarization condition was unpolarized/unpolarized. Solid curves represent an isotropic solid sphere with the indicated radius in the Rayleigh-Gans-Debye scattering.

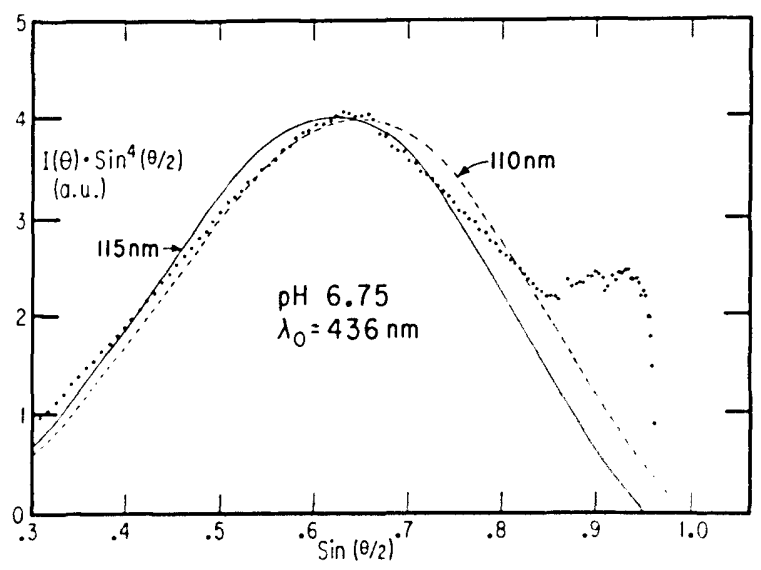

Figure 24. Same as figure 23 at $\mathrm{pH} 6.75$. 


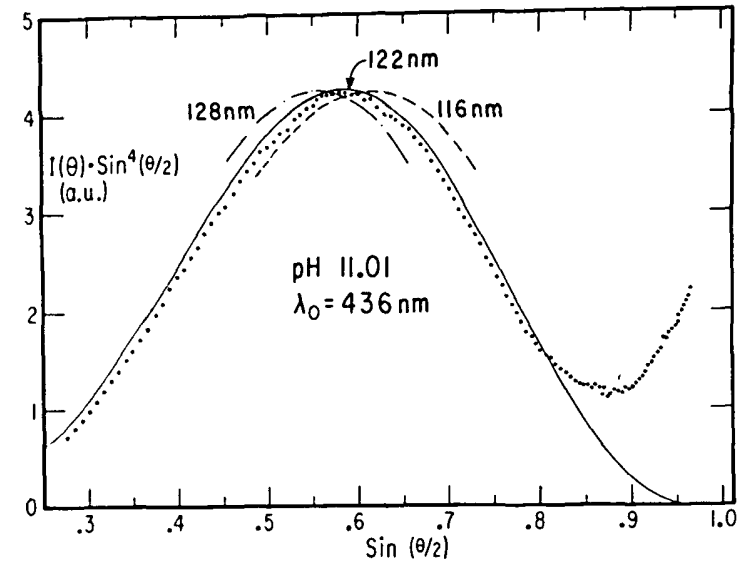

FIGURE 25. Same as figure 23 at $\mathrm{pH} 11.01$.

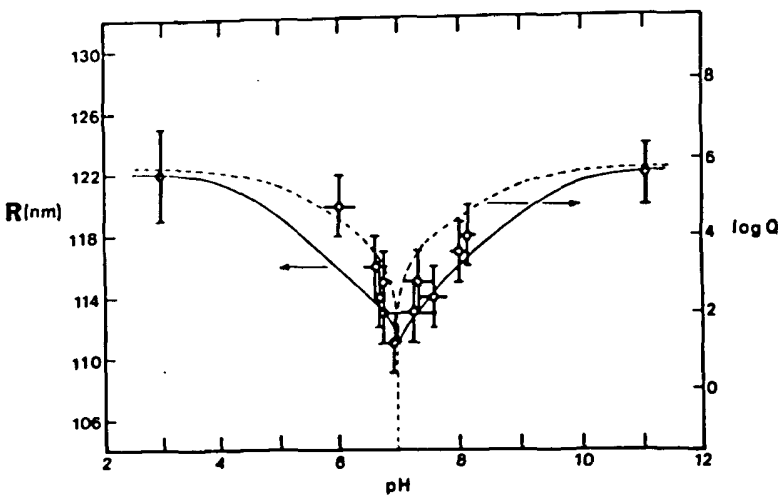

Figure 26. The particle radius versus $\mathrm{pH}$. The dashed curve represents the degree of ionization and the solid curve a model of simple polyelectrolyte effect for the shell layer.

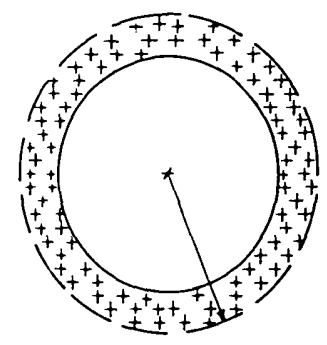

$\mathrm{pH} 3$

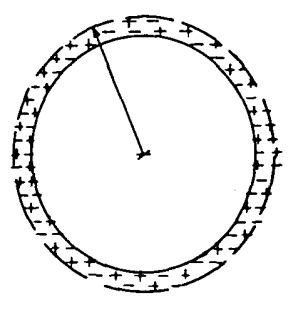

$\mathrm{pH} 7$

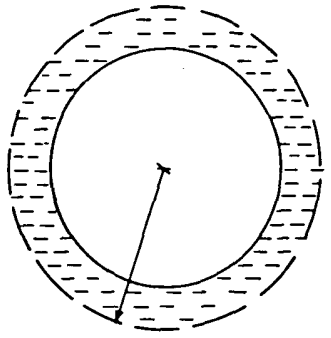

$\mathrm{pH} \mathrm{II}$

FIGURE 27. Schematic representation of $\mathrm{pH}$ induced swelling and deswelling of latex particles.

in the swollen state. Hence the latex particle dilation could easily be larger than what we report here. Unambiguous confirmation of the concentric sphere model is not possible with light scatterings alone. It might be possible to employ small angle neutron scattering [53] with perdeuterated core (with fully deuterated styrene monomer) and an appropriate $\mathrm{D}_{2} \mathrm{O} / \mathrm{H}_{2} \mathrm{O}$ mixture to contrast match the core and solvent such that the scattering due to the shell volume can be accentuated, whereby we could indeed measure the shell thickness dilation with $\mathrm{pH}$.

\section{b. Photoreceptor Disk Membrane Vesicles}

The vertebrate visual process involves complex sequences of events in transducing photochemical energy to electrical energy [54-56]. A vast literature exists concerning the role of the visual pigment membrane in this process $[57,58]$. Our goal has been to isolate disk membranes from vertebrate rod outer segment (ROS) as intact as possible [59] and focus on their static and dynamic structure relative to the photoreceptor function. I shall now discuss what we have learned about these membranes by isolating the disk membranes from ROS, swelling them into vesicles in hypotonic media and examining them in dilute suspensions by quasielastic and elastic light scatterings.

A typical homodyne power spectrum is shown in figure 28 and the spectral halfwidths obtained at different scattering angles are displayed in figure 29 . From these, we deduced the Stokes radius of $0.51 \pm 0.05 \mu \mathrm{m}$. If the vesicles were spherical in shape, then modelling them as spherical shells was quite reasonable because the bilayer thickness [60] of about $75 \mathrm{~nm}$ was negligibly small compared to $500 \mathrm{~nm}$ for the Stokes radius. Analogous to eq (9), one obtains from Eqs (5) through (8) in the limit of $p=q=0$,

$$
x^{2} P(\theta)=\sin ^{2} x
$$

for spherical shell. The results of elastic light scattering plotted according to eq (23) are shown in figure 30 . The equilibrium radius calculated from the Bragg condition of $Q R=n \pi / 2$, where the maxima are given by $n=$ odd integers and the minima by $n=$ even integers, is $0.48 \pm 0.06 \mu \mathrm{m}$. In 


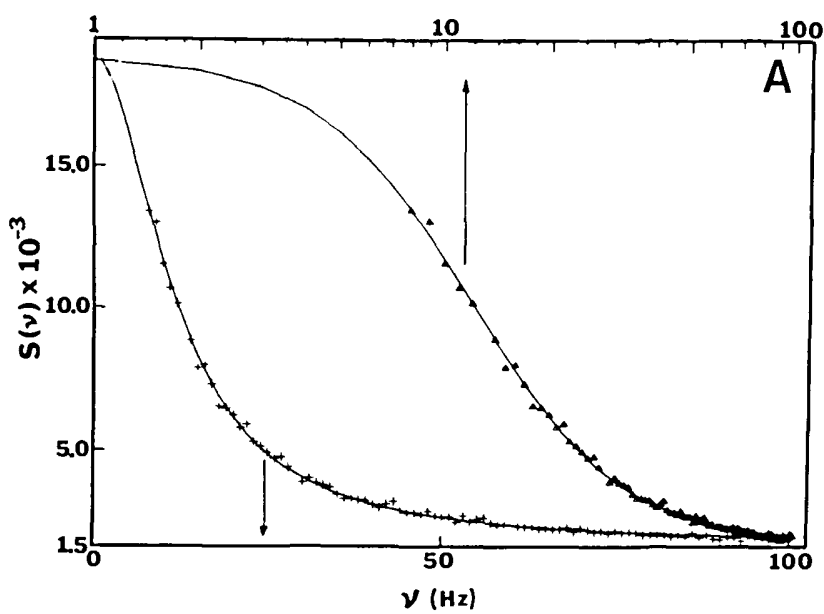

view of the agreement between the results of quasielastic and elastic light scattering, we take the spherical shell model for the vesicles to be valid in this particular suspending medium $[61,62]$.

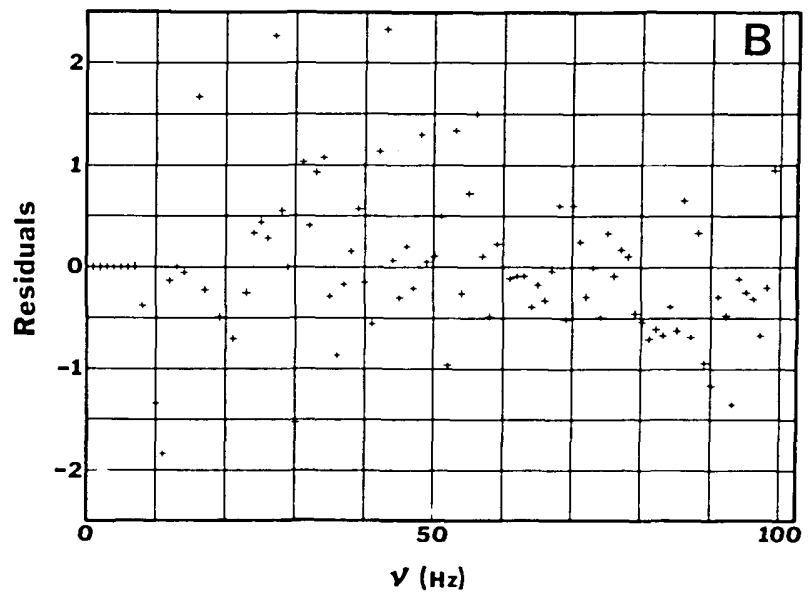

Figure 28. An example of homodyne power spectrum of the scattered light from photoreceptor disk membrane vesicles at $50^{\circ}$ scattering angle. Each solid curve in the upper figure represents a single Lorentzian profile with a halfwidth $\Delta v_{1 / 2}$ of $16 \mathrm{~Hz}$. In the lower figure, the normalized residuals of fitting to the single Lorentzian is plotted against frequency $v$.

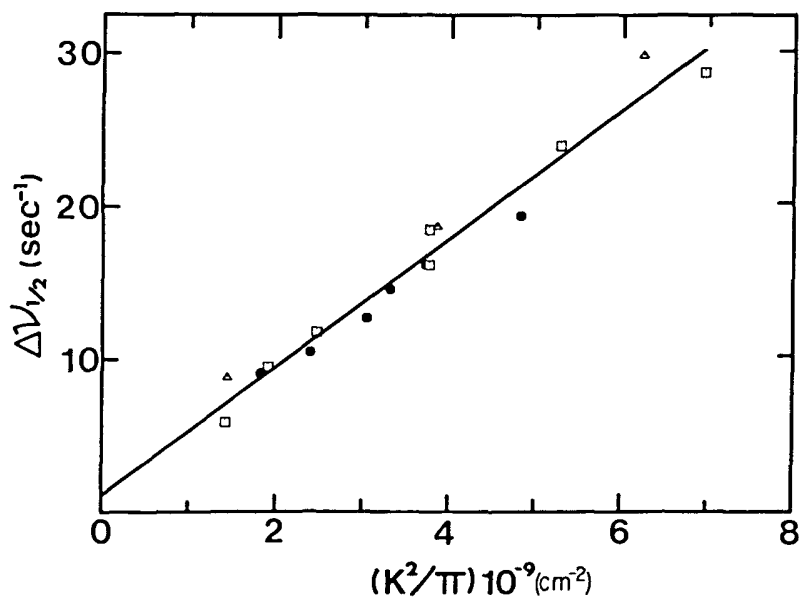

Ficure 29. $\Delta v_{1 / 2}$ versus $k^{2} / \pi$. Three independently prepared samples are distinguished by different symbols.

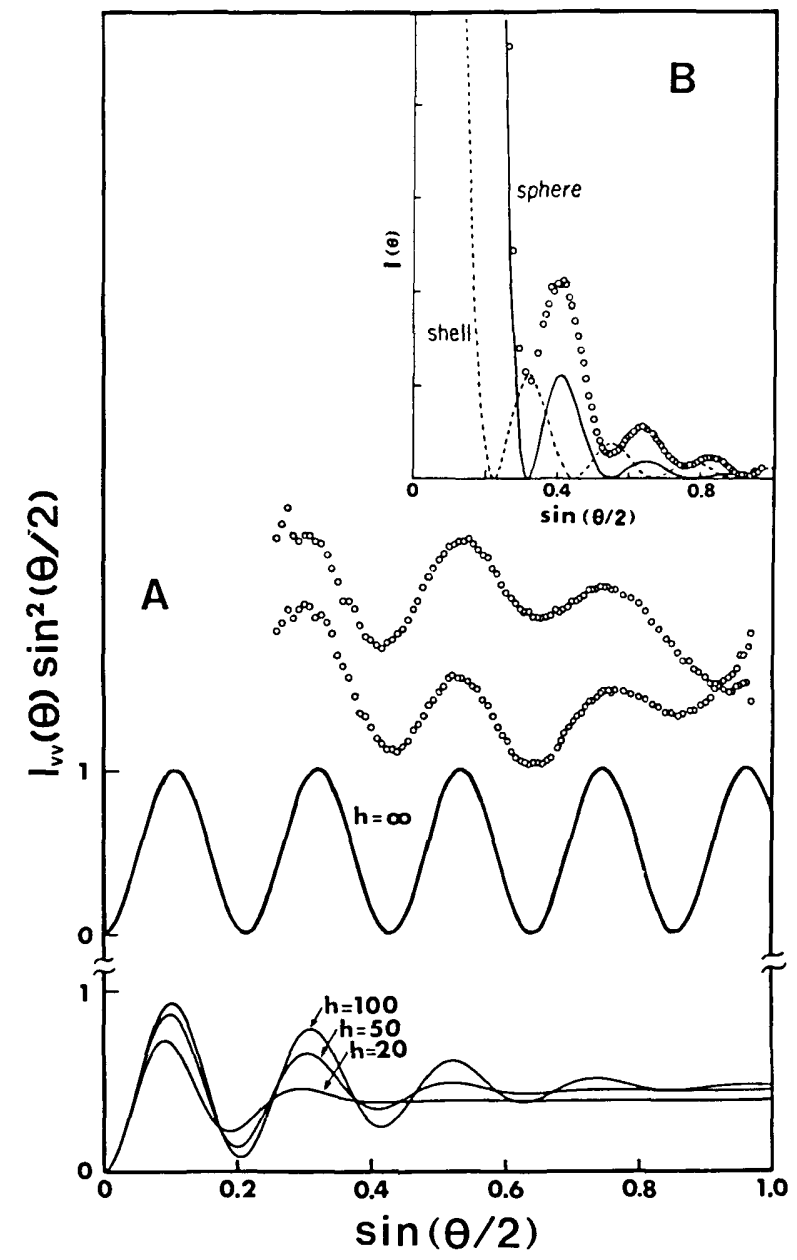

FIGURE 30A. The scattered intensity modulation profiles of two independently prepared vesicle suspensions of ROS membranes are displayed by plots of $I_{v v}(\theta) \sin ^{2}(\theta / 2)$ against $\sin (\theta / 2)$. The two sets are vertically shifted to exhibit the reproducibility of the extrema positions. The solid curves $x^{2} P(x)$ or $\left(Q R_{c}\right)^{2}<P(\theta)>$ is plotted against $\sin (\theta / 2)$. The parameters used for the theoretical profiles are $R=0.48 \mu \mathrm{m}$ for monodisperse shells $(h=\infty)$ and $R_{w}=0.48 \mu \mathrm{m}$ for polydisperse shells of the indicated distribution index $h$. Zimm-Schulz distribution function is used for the number fraction of shells with radius $R$ as

$$
f_{n}(R)=\frac{1}{h !}\left(\frac{h+1}{R_{w}}\right)^{h+1} R^{h} \exp \left[-\frac{(h+1) R}{R_{w}}\right]
$$

where $R_{w}$ is the weight average radius.

FICURE 30B. Comparison of the intensity profiles of solid sphere (solid) and sperical shell (dashed) is given by two theoretical curves; the experimental data for polystyrene latex sphere $(0.45 \mu$ m radius) are represented by the circles. Here, the plot is $I(\theta)$ versus $\sin (\theta / 2)$ unlike those in (30a). 
Upon changing the osmotic pressure of the suspending medium by an impermeable second solute, e.g., sucrose, we observe that the vesicles deform by deswelling, which is caused by the chemical potential gradient of the principal permeable component, namely $\mathrm{H}_{2} \mathrm{O}$. From the osmotic deformation behavior of the vesicles we were able to estimate the lateral compressive modulus of the membrane bilayer [63]. This turned out to be around $3 \times 10^{3} \mathrm{~Pa}$.

Since the membrane vesicles respond to the chemical potential gradient of water across the bilayer, their deformation behavior can be used to probe the permeability of ionic components including the hydrogen ion. Elastic light scattering studies of the vesicle shape have shown that the hydrogen ion is completely permeable within the $\mathrm{pH}$ range of 6-8 [64]. This is shown in figure 31 where the scattering profile of the spherical shell shape of the vesicles is hardly affected.

The binding of $\mathrm{Ca}^{+2}$ on vesicles was then studied by electrophoretic light scattering [65]. The Doppler shift spectra all at $7^{\circ}$ scattering angle and $20^{\circ} \mathrm{C}$ at a constant ionic strength of $1 \mathrm{mM}$ are displayed in figure 32 . The corresponding electrophoretic mobility profile at different $\mathrm{Ca}^{+2}$ concentrations is shown in figure 33 where the solid curve is drawn with a two-binding sites model. It can be represented by

$$
r / C=K_{1} C\left(n_{1}-r\right)+n_{2} K_{2}\left(\frac{1+K_{1} C^{2}}{1+K_{2} C}\right)
$$

where $r$ is the number average bound $\mathrm{Ca}^{+2}$ per vesicle, $n_{1}$ and $K_{1}$ are respectively the number of high affinity, a second order cooperative binding sites and the corresponding binding constant, and $n_{2}$ and $K_{2}$ are the other set of constants for low affinity, a first order non-cooperative binding. By replotting the data in figure 32, a Scatchard plot shown in figure 34 results, from which we estimate $n_{1}=$ $(1.4 \pm 0.1) \times 10^{4}$ and $K_{1}=(7 \pm 2) \times 10^{10} M^{-2}$ while $n_{2}$ and $K_{2}$ are subject to a good deal of uncertainty.

\section{c. Binary Nematic Solutions}

Here, our interest was focused on examining how the phase behavior [66] and dynamic twist modes [67] were affected by mixing of non-nematogens (biphenyl and benzene) to a thermotropic nematic liquid crystal, methoxy benzylidene butyl-aniline (MBBA). The scattering technique is the FDS method where the director of the nematic system is oriented parallel to the polarization of incident light while that of scattered light is perpendicular to the director axis [68]. Two examples of the FDS power spectra are displayed in Fig 35. With use of the twist viscosity of Gahwiller [69], we were able to determine the twist elastic constant of pure MBBA as a function of the reduced temperature. This is shown in figure 36 . Incorporating the concentration de- pendence of a non-nematogen to that of temperature, we could deduce the mapping of the twist diffusivity coefficient over the entire binary nematic phase region. In figure 37, such a mapping is displayed where the non-nematogen is biphenyl [68]. Fearing that this might have been unique to the biphenyl-MBBA system, benzene-MBBA nematic solution was also examined [70]. The result, as shown in figure 38 , indicates that such a behavior is not restricted to the biphenyl-MBBA system.

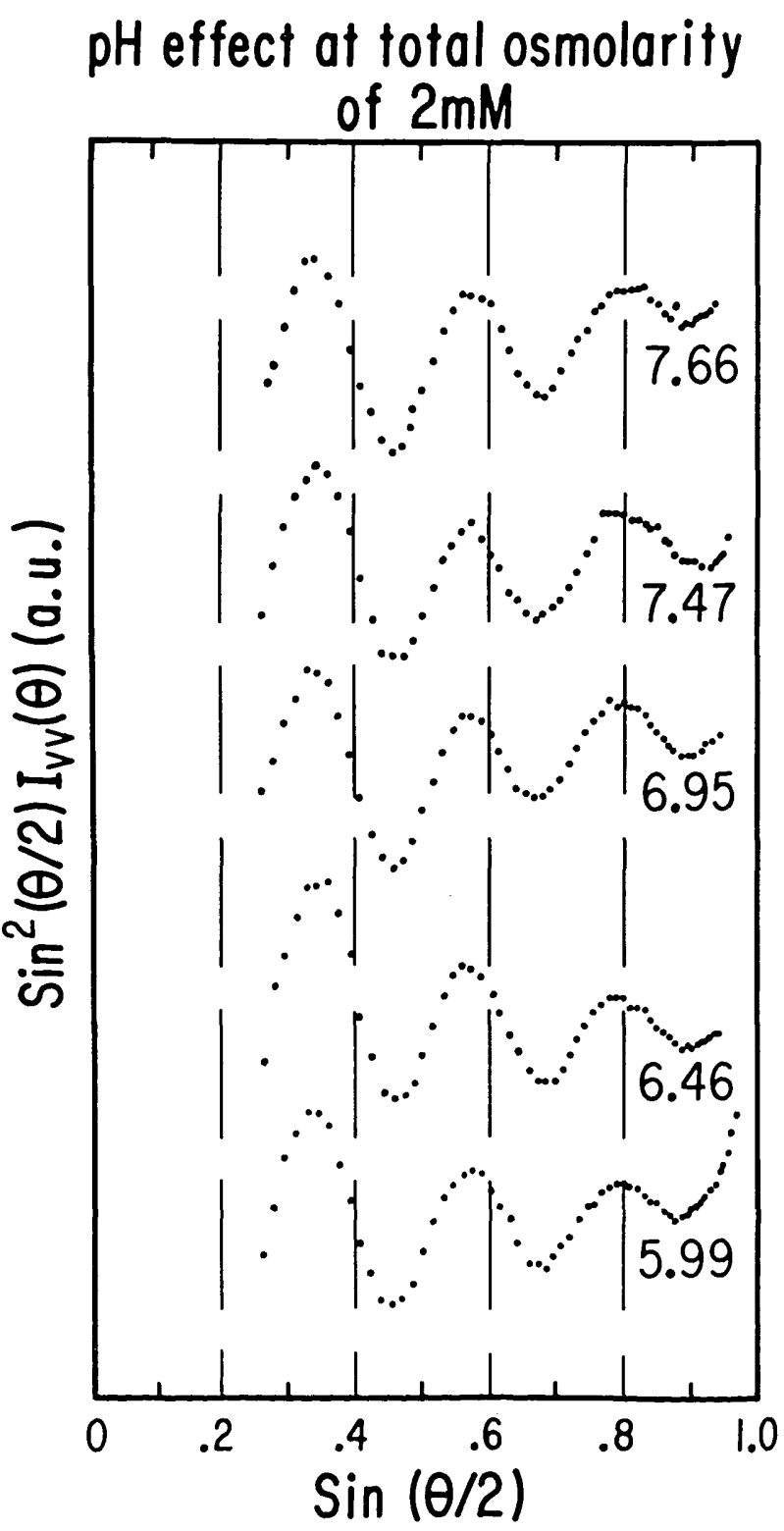

FIGURE 31. Light scattering intensity profiles of the vesicles at different $\mathrm{pH}$ to show that hydrogen ion is completely permeable to the membranes within the $\mathrm{pH}$ range studied. Profiles give no indication of the osmotic deformation which is clear evidence for shape invariability. 


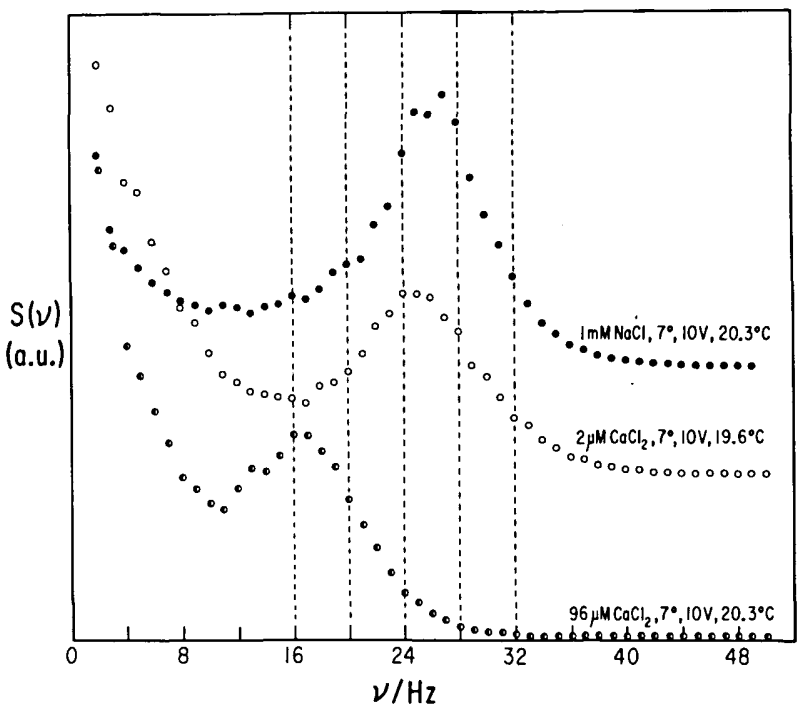

Figure 32. Three heterodyne spectra of electrophoretic light scattering, all at $7^{\circ}$ scattering angle and $10 \mathrm{~V}$ peak-to-peak $(27 \mathrm{~V} / \mathrm{cm})$ at three different $\mathrm{Ca}^{+2}$ concentrations at $1 \mathrm{mM}$ ionic strength solution

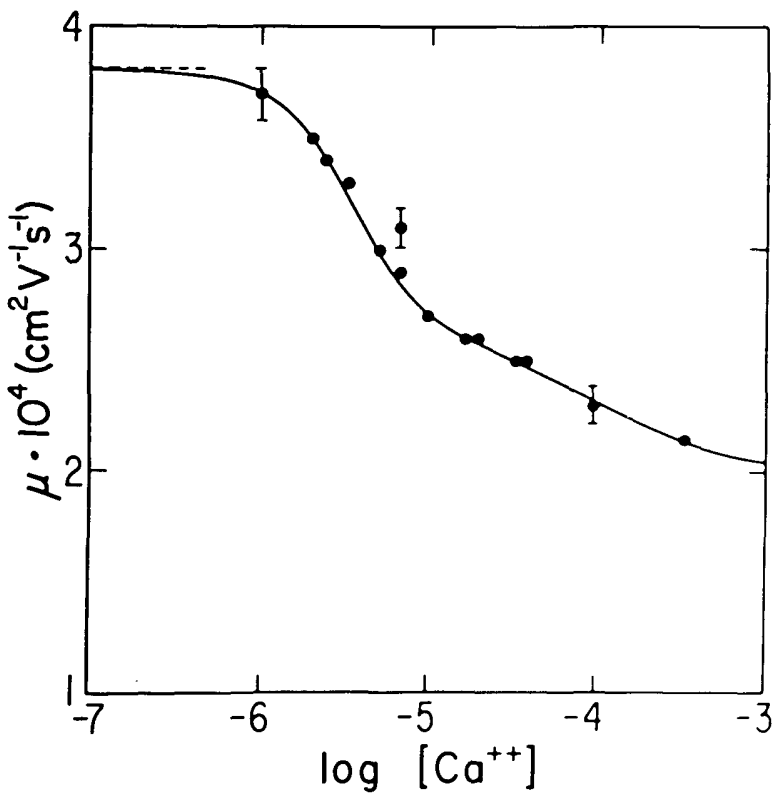

Figure 33. The electrophoretic mobility, corrected to $20^{\circ} \mathrm{C}$ in water, is plotted against $\mathrm{Ca}^{+2}$ concentration in log scale. Error bars apply to every data point although only three are shown. The solid curve is drawn according to the model discussed in the text.

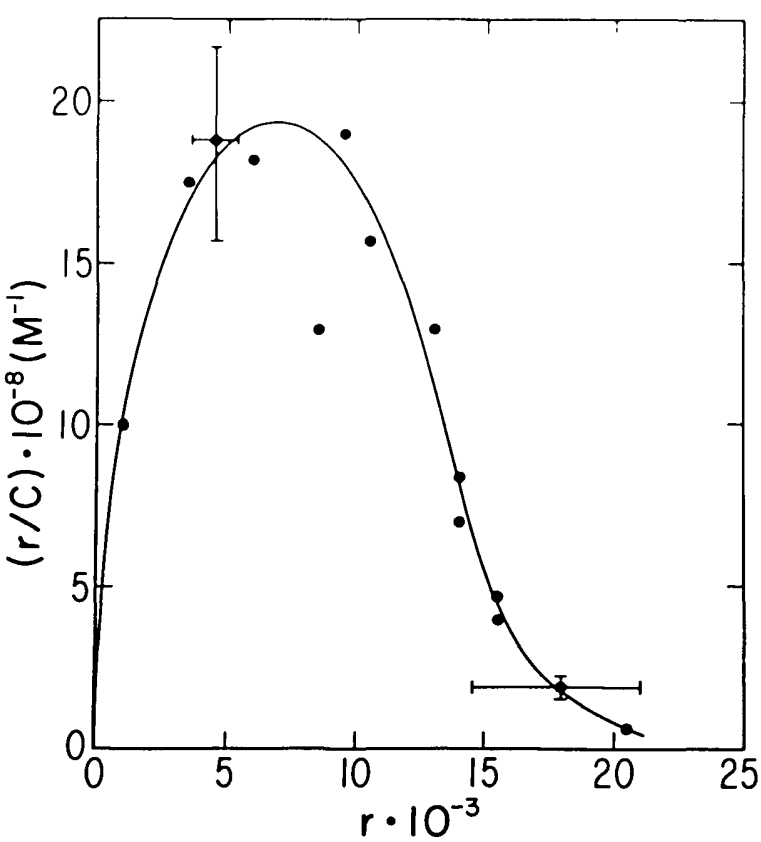

FIGURE 34. A Scatched plot where the number average degree of binding per unit ligand concentration $r / C$ is plotted against the degree of binding $r$. Error bars apply to every data point although only two are shown. Solid curve is drawn according to the model discussed in the next while dashed line is drawn by ignoring lower five points in $r$. The intercept with the abscissa of the dashed line gives the apparent first order binding site number $n_{1}$ and its slope the apparent first order dissociation constant $K_{1}^{-1}$.

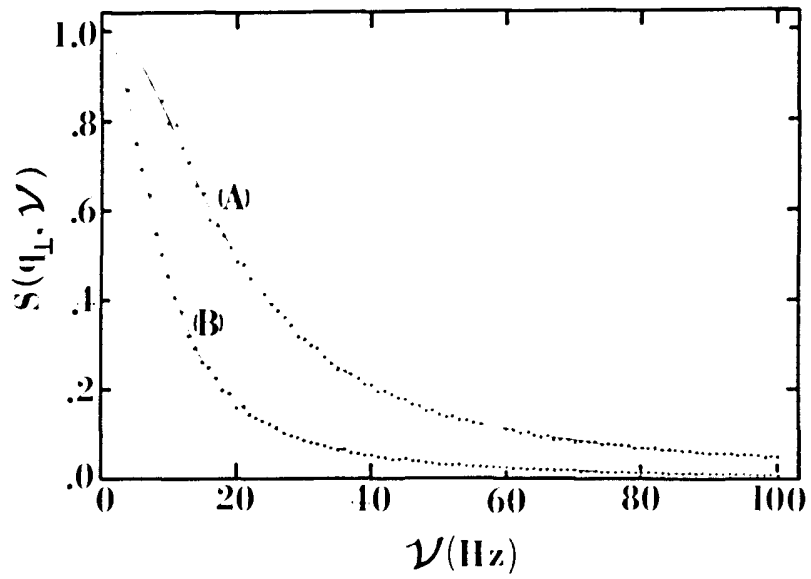

Ficure 35. Heterodyne power spectra of pure $\mathrm{MBBA}$ at $36.90^{\circ} \mathrm{C}, \Delta v_{1 / 2}=$ $30.1 \pm 0.1 \mathrm{~Hz}$ (upper) and at $44.95^{\circ} \mathrm{C}, \Delta v_{1 / 2}=6.9 \pm 0.6 \mathrm{~Hz}$ (lower). 


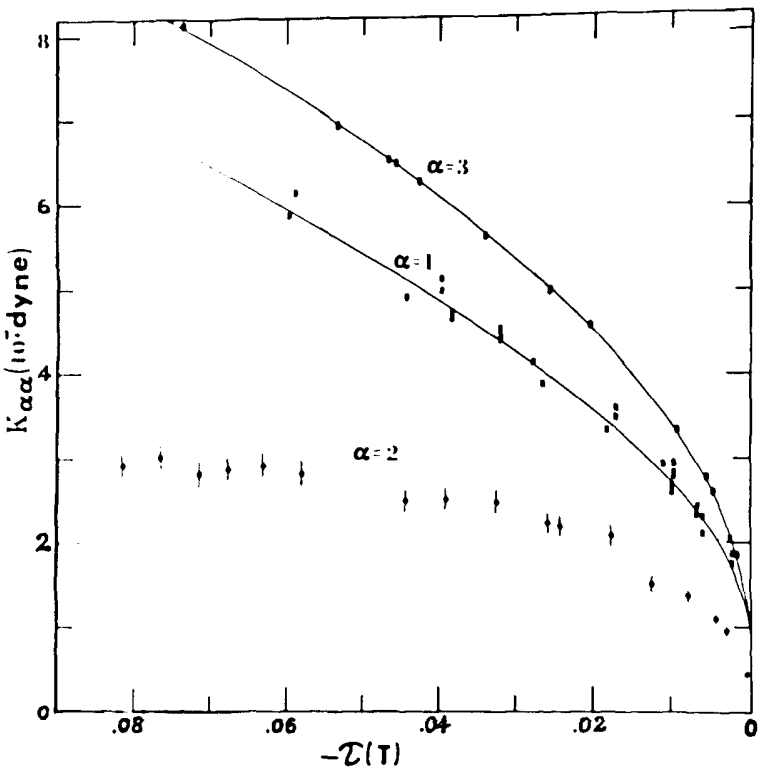

FICURE 36. The elastic constants of pure MBBA versus the reduced temperature $\pi(T)$. The bend $(a=3)$ and splay $(a=1)$ elastic constants are from the work of Haller and the twist constant $(a=2)$ is ours with use of the twist viscosity of Gahwiller.

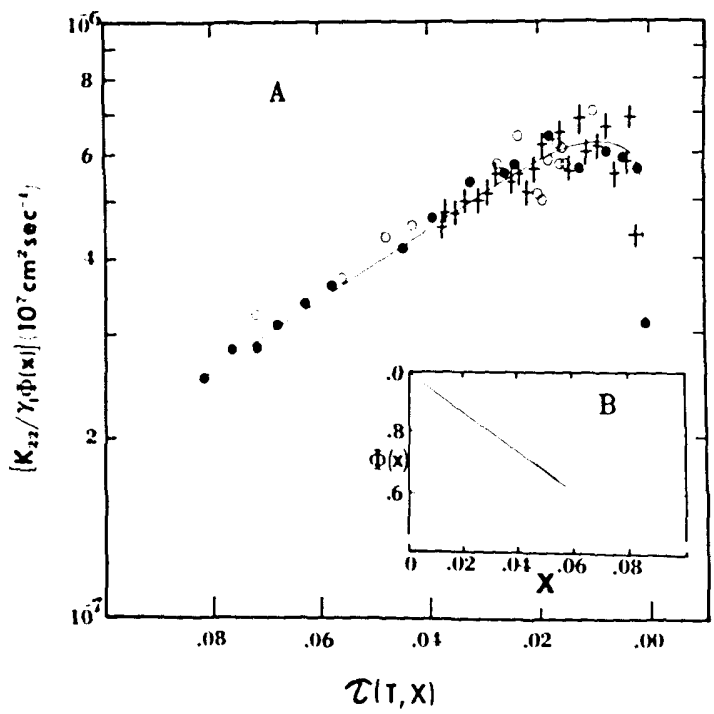

Figure 37A. Composition reduced twist diffusivity coefficient $K_{22} / \gamma_{1} \phi(X)$ versus the reduced temperature $T(T, X) \equiv T-T_{N 1}(X) / T_{n 1}(X)$, where $T_{n 1}(X)$ $=(0)(1-250 X)$ as observed from the phase diagram. The filled circles are from temperature scan of pure MBBA, the open circles are from temperature scan of 0.081 mole fraction biphenyl solution and the crosses $(+)$ are from composition scan at $23^{\circ} \mathrm{C}$.

FIGURE 37B. $\Phi(X)$, the composition dependent factor of the twist diffusivity coefficient at constant reduced temperature versus mole fraction of biphenyl.

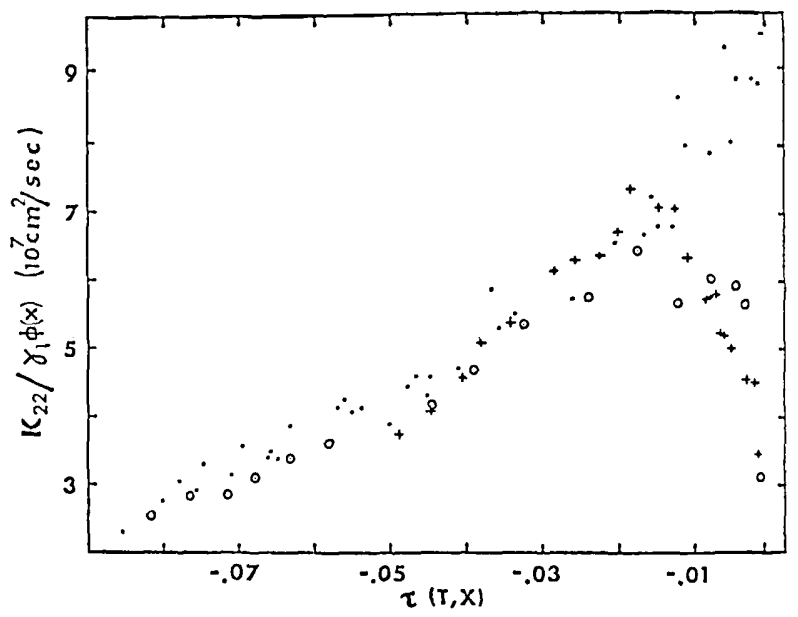

Figure 38. Same as figure 37 for the MBBA-benzene system.

\section{d. Intramolecular Chain Dynamics of Isotactic Polystyrene}

A typical FDS power spectrum [46] of an isotactic polystyrene sample $\left(M w=3.5 \times 10^{6},\left\langle s^{2}\right\rangle^{1 / 2}=1270 \AA\right)$ in tetrahydrofuran at $4.0 \mathrm{mg} / \mathrm{mL}$ is shown in Fig 39 . All spectra were analyzed according to eq (21) with the Zimm spacing [71] of relaxation times up to five relaxation modes. Truncation beyond the 6 th mode was called for due to the chosen bandwidth of $2 \mathrm{kHz}$ whereby the higher modes were obscured by the shot-noise level. Spectral analysis was effected by a 3-parameter fitting routine; the parameters were the spectral intensity, shot-noise level and the terminal relaxation time $\tau_{1}$. By fixing the spacing to that of Zimm type, what we extract from the experiment is $\tau_{1}$, the slowest relaxation time of internal normal modes. Because all measurements were performed at finite concentrations, we had to extrapolate the data to infinite dilution. This is effected by plotting $\tau_{1}$ against relative viscosity $\eta_{r}$ of polymer solution and extrapolating to $\eta_{r}=1$. Such a plot is shown in figure 40 from which $\tau_{1}$ at infinite dilution is determined as (1.2-1.5) $\times 10^{-4}$ sec with an experimental uncertainty of $\pm 30 \%$. The theoretical value of $\tau_{1}$ for the polymer sample is $1.5 \times 10^{-4} \mathrm{sec}$. Within the experimental error the two are in agreement.

\section{Concluding Remarks}

It is my hope that the readers of this report would be as excited as I have been about the potentials of light scattering techniques and have some appreciation for what kinds of problems can be tackled. I must again emphasize that this is intended only as a sketchy review of what my students and $I$ have been able to do and not as an up-to-date review of the field. I have cited several excellent reviews in the text. 


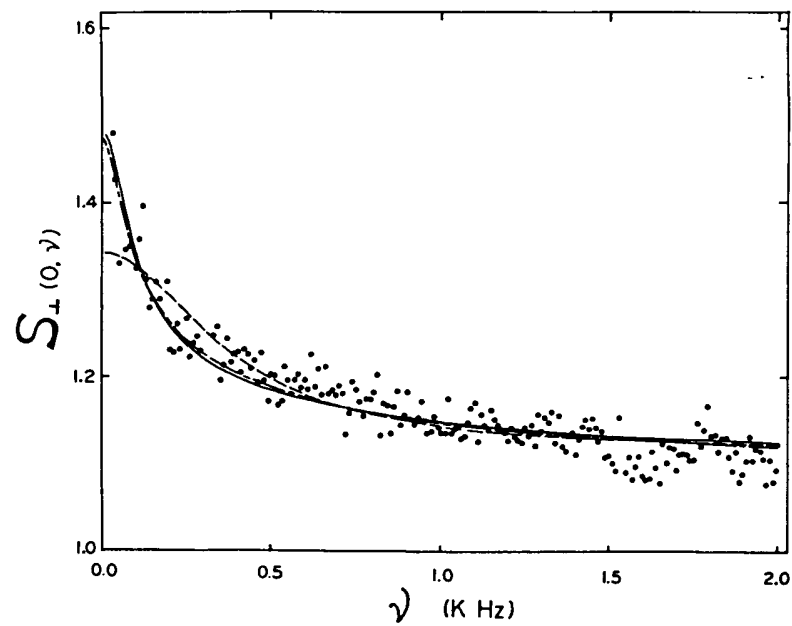

FIGURE 39. FDS power spectrum of isotactic polystyrene (M.W. $3.5 \times 10^{6}$ ) in THF at $C=4.0 \mathrm{mg} / \mathrm{mL}$. The solid curve is for a single Lorentzian fit and the dashed curve is for $\mathrm{Zimm}$ fit with the first five relaxation times (Z5).

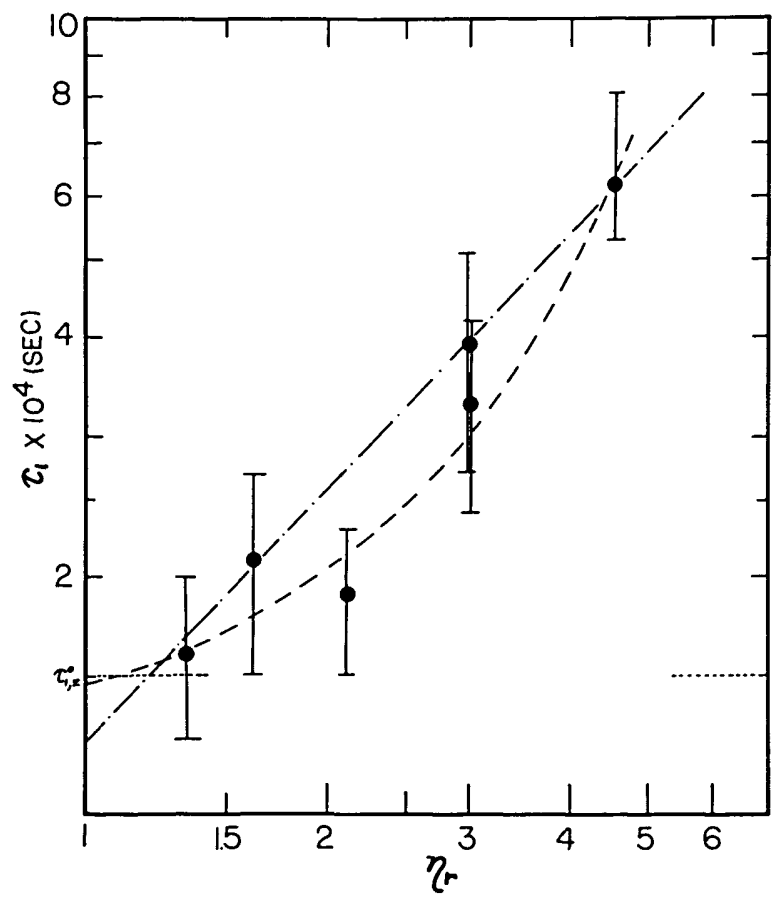

FICURE 40. The terminal relaxation time deduced from the $\mathbf{Z 5}$ fit of the FDS power spectra of isotactic polystyrene in THF is plotted against rela. tive viscosity at different concentrations $(0.65 \mathrm{mg} / \mathrm{mL}-4.0 \mathrm{mg} / \mathrm{mL}$. Error bars define $95 \%$ confidence limit of the fit and the theoretical value of the Zimm model is designated as $\tau_{i, \text {, in the ordinate. }}^{0}$ in
The work presented here is the result of efforts by many former and current students and associates of mine with whom I have been fortunate enough to be associated in the past nine years, and I thank each and every one of them by name: Charles C. Han, Steven A. Shaya, Alex R. Sluzas, William F. Hoffman, Takashi Norisuye, George B. Caflisch, Eric J. Amis, David M. Piatt, Jeffrey A. Wesson, Taihyun Chang, Deborah A. Davenport, Jeffrey L. Nelson, Edward D. Erickson, Dong M. Pham, and Dan J. Wendt.

Support by the National Institutes of Health through EY 01483 and a Biomedical Research Support Grant administered through the Graduate School of the University of Wisconsin-Madison are gratefully acknowledged.

\section{References}

[1] Cummins, H. Z. and Swinney, H. L. (1970) Prog. Opt. 8, 133.

[2] Pecora, R. (1972) Annu. Rev. Biophys. Bioeng. 1, 257.

[3] Chu, B. (1974) Laser Light Scattering (Academic, New York).

[4] Berne, B. J. and Pecora, R. (1976) Dynamic Light Scattering (Wiley, New York).

[5] Cummins, H. Z. and Pike, E. R., ed. (1974) Photon Correlation and Light Beating Spectroscopy (Plenum Press, New York).

[6] Zimm, B. H., Stein, R. S. and Doty, P. (1945) Polym. Bull. 1, 90.

[7] Stacey, K. A. (1956) Light Scattering in Physical Chemistry (Academic, New York).

[8] Kerker, M. (1969) The Scattering of Light and Other Electromagnetic Radiation (Academic, New York).

[9] Bloomfield, V. A., Crothers, D. M. and Tinoco, I., Jr. (1974) Physical Chemistry of Nucleic Acids (Harper and Row, New York), p. 187.

[10] Zimm, B. H. (1948) J. Chem. Phys. 16, 1099.

[11] Debye, P. (1944) J. Appl. Phys. 15, 338.

[12] Kerker, M. (1969) The Scattering of Light and Other Electromagnetic Radiation (Academic, New York), pp. 482-485.

[13] Oster, G. and Riley, D. P. (1952) Acta Crystallogr. 5, 1.

[14] Pecora, R. and Aragon, S. R. (1974) Chem. Phys. Lipids 13, 1.

[15] Saito, N. and Ikeda, Y. (1951) J. Phys. Soc. Japan 6, 305.

[16] Chang, T., Pham, D. M., Piatt, D. M. and Yu, H., to be published.

[17] Heller, W. and Pugh, T. L. (1957) J. Colloid Sci. 12, 294.

[18] Shaya, S.A., Han, C. C. and Yu, H. (1974) Rev. Sci. Instrum. 45, 280.

[19] Ware, B. R. and Flygare, W. H. (1971) Chem. Phys. Lett. 12, 81.

[20] Uzgiris, E. E. (1972) 0pt. Commun. 6, 55.

[21] Ware, B. R. and Flygare, W. H. (1972) J. Colloid Interface Sci. 39, 670.

[22] Bennett, A. J. and Uzgiris, E. E. (1973) Phys. Rev. A 8, 2662.

[23] Uzgiris, E. E. (1974) Rev. Sci. Instrum. 45, 74.

[24] Uzgiris, E. E. and J. H. Kaplan (1974) Rev. Sci. Instrum. 45, 120.

[25] Ware, B. R. (1974) Advan. Coll. Interface Sci. 4,1.

[26] Haas, D. D. and Ware, B. R. (1976) Anal. Biochem. 74,175.

[27] Flygare, W. H. and Ware, B. R. (1976) in Molecular Electro-optics, 0'Konski, C. T., Ed. (Marcel Dekker, New York), p. 544.

[28] Mohan, R., Steiner, R. and Kaufman, R. (1976) Anal. Biochem. 79, 506.

[29] Josefowicz, J. and Hallet, F. R. (1975) Appl. Opt. 14, 740.

[30] Schlessinger, B. S. (1958) J. Phys. Chem. 62, 916.

[31] Alberty, R. A. (1948) J. Chem. Edu. 25, 426. 
[32] Longsworth, L. G. and Jacobsen, C. F.(1949) J. Phys. Chem. 53, 126.

[33] Caflisch, G. B., Norisuye, T. and Yu, Hyuk, (1980) J. Colloid Interface Sci. 76, 174.

[34] Henry, D. C. (1931) Proc., Roy. Soc. (London) A133, 106.

[35] See for a recent survey, Shaw, D. J.(1969) Electrophoresis (Academic, New York).

[36] Tiselius, A. and Svensson, H. (1940) Trans. Faraday Soc. 36, 16.

[37] Alexander, A. E. and Johndon, P. (1949) Colloid Science (Oxford University Press, London), p. 742.

[38] Cannan, R. K., Palmer, A. K. and Kibrick, A. (1942) J. Biol. Chem. 142, 803.

[39] Abramowitz, M. and Stegun, I. A. (1964) Handbook of Mathematical Functions, NBS Applied Mathematics Series 55 (U.S. Government Printing Office, Washington, D.C.), p.555.

[40] Baldwin, R. L., Gosting, L. J., Williams, J. W. and Alberty, R.A. (1955) Discussions Faraday Soc. 20, 13.

[41] Wada, A., Suda, N., Tsuda, T. and Soda, K. (1968) J. Chem. Phys. 50, 31.

[42] Wada, A., Soda, K., Tanaka, T. and Suda, N. (1970) Rev. Sci. Instrum. 41, 845 .

[43] Schurr, J. M. and Schmitz, K. S.(1973) Biopolymers 12, 1021.

[44] Schmitz, K. S. and Schurr, J. M. (1973) Biopolymers 12, 1543.

[45] Norisuye, T. and Yu, H. (1978) J. Chem. Phys. 68, 4038.

[46] Han, C. C. and Yu, H. (1974) J. Chem. Phys. 61, 2650.

[47] Yen, S.P.S., Rembaum, A., Molday, R. S. and Dreyer, W. J. (1976) Emulsion Polymerization, Pirma, I. and Gordon, J. eds., ACS Symposium Series, Vol. 24 (American Chemical Society, Washington, DC), p. 236.

[48] Molday, R. S., Dreyer, W. J., Rembaum, A. and Yen, S.P.S. (1975) J. Cell. Biol. 64, 75.

[49] Jacobson, B. S. and Branton, D. (1977) Science 195, 302.
[50] Vanderhoff, J. W. and van den Hull, H. J. (1973) J. Macromol Sci. Chem. A7, 677.

[51] Homola, J. and James, R. 0. (1977) J. Colloid Interface Sci. 59, 123.

[52] Piatt, D. M., Wesson, J. A., Caflisch, G. B., Nelson, J. L. and Yu, H., to be published.

[53] See for a recent review, Maconnachie, A. and Richards, R. W. (1978) Polymer 19, 739.

[54] Wald, G. (1968) Science 162, 230.

[55] Rodieck, R. (1973) The Vertebrate Retina (Freeman, San Francisco).

[56] Kliger, D. S. and Menger, E. L. (1975) Acc. Chem. Res. 8, 81.

[57] Hagins, W. A. (1972) Annu. Rev. Biophys. Bioeng. 1, 131.

[58] Abrahamson, E. W. (1975) Acc. Chem. Res. 8, 101.

[59] Amis, E. J., Davenport, D. A. and Yu, H., Anal. Biochem., in press.

[60] Worthington, C. R. (1973) Exp. Eye Res. 17, 487.

[61] Norisuye, T., Hoffman, W. F. and Yu, H. (1976) Biochemistry 15, 5678.

[62] Hoffman, W. F., Norisuye, T. and Yu, H. (1976) Biochemistry 16, 1273.

[63] Norisuye, T. and Yu, H. (1977) Biochim. Biophys. Acta 471, 436.

[64] Amis, E. J., Wendt, D. A., Erickson, E. D. and Yu, H., Biochim. Biophys. Acta, in press.

[65] Caflisch, G. B. (1979) Ph.D. Thesis, University of Wisconsin, Madison, Wisconsin; Piatt, D. M., Caflisch, G. B. and Yu, H., to be published.

[66] Shaya, S. A. and Yu, H.(1975) J. Phys. (Paris) C1(3), 59.

[67] deGennes, P. G. (1974) The Physics of Liquid Crystals (Oxford, New York).

[68] Shaya, S. A. and Yu, H. (1975) J. Chem. Phys. 63, 221.

[69] Gahwiller, Ch. (1973) Mol. Cryst. Liquid Cryst. 20, 301.

[70] Sluzas, A. R. (1975) Ph.D. Thesis, University of Wisconsin, Madison, WI.

[71] Zimm, B. H. (1956) J. Chem. Phys. 24, 269. 